\title{
NEAMS-Funded University Research in Support of TREAT Modeling and Simulation, FY15
}

Mark DeHart Idaho National Laboratory

Zander Mausolff and Sedat Goluoglu University of Florida

Zachary Prince and Jean Ragusa

Texas A\&M University

Carl Haugen, Matt Ellis, Benoit Forget, Kord Smith Massachusetts Institute of Technology

Anthony Alberti and Todd Palmer Oregon State University

September 2015 Idaho National Laboratory 


\section{DISCLAIMER}

This information was prepared as an account of work sponsored by an agency of the U.S. Government. Neither the U.S. Government nor any agency thereof, nor any of their employees, makes any warranty, expressed or implied, or assumes any legal liability or responsibility for the accuracy, completeness, or usefulness, of any information, apparatus, product, or process disclosed, or represents that its use would not infringe privately owned rights. References herein to any specific commercial product, process, or service by trade name, trade mark, manufacturer, or otherwise, does not necessarily constitute or imply its endorsement, recommendation, or favoring by the U.S. Government or any agency thereof. The views and opinions of authors expressed herein do not necessarily state or reflect those of the U.S. Government or any agency thereof. 


\title{
NEAMS-Funded University Research in Support of TREAT Modeling and Simulation, FY15
}

\author{
Mark DeHart \\ Idaho National Laboratory \\ Zander Mausolff and Sedat Goluoglu \\ University of Florida \\ Zachary Prince and Jean Ragusa \\ Texas A\&M University \\ Carl Haugen, Matt Ellis, Benoit Forget, Kord Smith \\ Massachusetts Institute of Technology \\ Anthony Alberti and Todd Palmer \\ Oregon State University
}

September 2015

Idaho National Laboratory

Reactor Physics Analysis and Design Dept.

Idaho Falls, Idaho 83415

http://www.inl.gov

Prepared for the

U.S. Department of Energy

Office of Nuclear Energy

Under DOE Idaho Operations Office

Contract DE-AC07-05ID14517 


\title{
NEAMS-Funded University Research in Support of TREAT Modeling and Simulation, FY15
}

\author{
Mark DeHart ${ }^{\mathrm{a}}$, Zander Mausolff ${ }^{\mathrm{b}}$, Sedat Goluoglu ${ }^{\mathrm{b}}$, Zachary Prince $^{\mathrm{c}}$, Jean Ragusa ${ }^{\mathrm{c}}$, \\ Carl Haugen ${ }^{\mathrm{d}}$, Matt Ellis ${ }^{\mathrm{d}}$, Benoit Forget ${ }^{\mathrm{d}}$, Kord Smith ${ }^{\mathrm{d}}$, Anthony Alberti ${ }^{\mathrm{e}}$, Todd Palmer ${ }^{\mathrm{e}}$ \\ ${ }^{a}$ Idaho National Laboratory \\ ${ }^{\mathrm{b}}$ University of Florida \\ ${ }^{\mathrm{c}}$ Texas A\&M University \\ ${ }^{\mathrm{d}}$ Massachusetts Institute of Technology \\ ${ }^{\mathrm{e}}$ Oregon State University
}

\begin{abstract}
This report summarizes university research activities performed in support of TREAT modeling and simulation research. It is a compilation of annual research reports from four universities: University of Florida, Texas A\&M University, Massachusetts Institute of Technology and Oregon State University. The general research topics are, respectively, (1) 3-D time-dependent transport with TDKENO/KENO-VI, (2) implementation of the Improved Quasi-Static method in Rattlesnake/ MOOSE for time-dependent radiation transport approximations, (3) improved treatment of neutron cross section scattering treatment and collapse within TREAT using OpenMC, and (4) steady state modeling of the minimum critical core of the Transient Reactor Test Facility (TREAT).
\end{abstract}

\subsection{INTRODUCTION}

Efforts completed to date in support of modeling and simulation of the Idaho National Laboratory (INL) Transient Reactor Test Facility (TREAT) under fast transient conditions have barely scratched the surface. INL has made significant progress in terms of coupled multi-physics methods for TREAT transient calculations but there is much more to learn and understand about the basic physics of the system, and to develop appropriate analysis approaches that will capture the dominant effects without focusing on unimportant aspects of the core. 
In light of this fact, INL has directed a portion of NEAMS funding toward applied $\mathrm{R} \& \mathrm{D}$ at the university level to complement research being performed at INL. This provides and opportunity for student support, development and growth, provides options for internship posting at INL, and allows word to be performed that in independent of research and development efforts ongoing within the INL reactor physics modeling and simulation team.

Four research projects were supported in FY15, aligned with INL TREAT modeling and simulation mission needs. These projects are very coarsely described by the following titles:

- 3-D Time-Dependent Transport with TDKENO/KENO-VI, University of Florida, Mr. Zander Mausolff and Prof. Sedat Goluoglu, - Implementation of the Improved Quasi-Static Method in Rattlesnake/MOOSE for Time-Dependent Radiation Transport Approximations, Mr. Zachary Prince and Prof. Jean Ragusa, Texas A\&M University, - Improved Treatment of Neutron Cross Section Scattering Treatment and Collapse within TREAT using OpenMC, Mr. Carl Haugen, Mr. Matthew Ellis, Prof. Benoit Forget and Prof. Kord Smith, Massachusetts Institute of Technology. - Steady State Modeling of the Minimum Critical Core of the Transient Reactor Test Facility, Mr. Anthony Alberti and Prof. Todd Palmer, Oregon State University.

The following sections of this report are summaries of the research work, prepared by the students listed above under the supervision of their academic mentors. Each section is independent and stands on its own. 


\subsection{3-D TIME-DEPENDENT TRANSPORT WITH TDKENO/KENO-VI}

TDKENO employs a hybrid methodology for solving the time dependent, threedimensional Boltzmann transport equation. The current version of TDKENO uses the Monte Carlo Transport code KENO V.a from the SCALE code system to calculate the flux shapes and fixed sources. The TREAT fuel elements are square blocks with the corners chamfered such that when four elements are put in a $2 \times 2$ array there is a square channel in the center for air flow. The dog-ear corners are approximated with KENO V.a. This approximation can be eliminated by including KENO-VI as one of the options in TDKENO. As such, TDKENO has been modified to enable use of KENO-VI (in addition to KENO V.a.), which has a geometry package that can be used to exactly model the TREAT core. KENOVI allows generalized geometry input that is not possible in KENO V.a inputs. In order to verify the changes, TDKENO has been run using both KENO V.a and KENO-VI. Three different test cases have been analyzed: the computational benchmark 16-A1 [1], a simple TREAT model, and a transient model (2855) from the M8CAL calibration tests performed in TREAT.

\subsection{DESCRIPTION OF TDKENO}

Full core transient analysis requires understanding how neutrons behave inside a reactor as time evolves. This is accomplished by solving the equation governing the time evolution of neutron population, known as the three-dimensional (3-D), time-dependent Boltzmann Transport equation. The neutron population allows factors dictating the power and safety of a core to be calculated. Approximations

typically used to understand neutron populations and the underlying physics, such as multi-group, multi-dimensional diffusion theory can fail in strongly absorbing regions and voided regions and require special treatments [2]. This has encouraged the development of codes such as TDKENO, which utilizes a hybrid method for solving the time dependent 3-D transport equation with explicit representation of delayed neutrons. 
In TDKENO the time variable is handled with the improved quasi-static method. This is denoted as a flux factorization method as the flux can be factored, as shown in Equation (2.1), where $\psi(r, E, \Omega, t)$ is the angular flux at position $\mathrm{r}$, energy $\mathrm{E}$ and time $\mathrm{t}, T(t)$ is the amplitude function and $\Phi(r, E, \Omega, t)$ is the flux shape function with weak time dependence.

$$
\psi(r, E, \Omega, t)=T(t) \cdot \Phi(r, E, \Omega, t)
$$

Uniqueness is imposed on this factorization by inserting a constant normalization factor throughout the transient [3]. TDKENO is considered a hybrid solution method, because the amplitude function is solved deterministically whereas the flux shape is calculated via a Monte Carlo method [3,4]. Calculation of the flux shape is computationally expensive, thus is only calculated when an update is required. However, the amplitude portion is solved many times over small time intervals.

To obtain the position, energy, and angle dependent neutron flux shape, the Monte Carlo code KENO from the SCALE package is employed. KENO may refer to either KENO V.a (simpler geometry) or KENO-VI (generalized geometry) as now both are available for use in TDKENO. Since this work focuses on the integration of KENO-VI with TDKENO we will describe KENOVI in detail. It should be noted that the only difference in KENO versions is how geometry is handled so the theoretical treatment applies to both. Before delving into KENO we will highlight another aspect of TDKENO's methodology, particularly pertaining to transient analysis.

To accurately determine the power, total yield and point kinetics parameters such as reactivity and effective delayed neutron fractions, a suitable feedback method is necessary. Values such as reactivity and effective delayed neutron fractions are not measured directly [2]. Rather, the periods of delayed or prompt supercritical 
systems is used to infer the reactivity. In addition, time lag of delayed neutrons allows better control of the form of transients. TDKENO incorporates feedback via quenching coefficients provided a priori [4]. The core temperature affects reactivity as well. To incorporate temperature-dependent cross sections TDKENO would need to be coupled to a code that provides the temperature distribution as a function of energy created in the core. At present this is not done and an approximation is used. The feedback mechanism that calculates the reactivity as a function of total energy produced in the core is implemented and described in equation (2) as:

$$
\rho_{f b}(t)=\sum_{i=0}^{3} a_{i} Y^{i}(t)
$$

where $\rho_{f b}(t)$ is the feedback reactivity, $a_{i}$ are the empirical coefficients for a third order polynomial and $Y(t)$ is the total yield in the core at time $t$ in MJ.

\subsection{CALCULATION FLOW}

In this Section we give a brief overview of the calculation flow of TDKENO. The initial step in a given problem is to perform a steady state adjoint calculation using KENO. Note that this can be turned off if the adjoint flux is already known and provided in the working directory. The steady state adjoint flux is used as a weighting function in the IQS method in calculating the point kinetics parameters. Similarly, the forward calculation is done with KENO and determines the initial flux shape, the effective multiplication factor, and constraint integral. To determine the initial values of the point kinetics parameters and the flux amplitude the subroutine RHO and PTKIN are called by TDKENO, respectively. If the user wishes to incorporate thermal feedback into the system the subroutine FEEDBACK is called to calculate the adiabatic heat-up model. The delayed neutron distribution and cumulative distribution functions needed for the flux shape derivative are done by CDELAY. Finally, TDKENO calls KENO to 
perform the flux shape calculation of the perturbed system. The process outlined above is repeated until the desired amount of flux shapes are computed or the desired cutoff run time is reached. Once the sequence is terminated the subroutine POWER calculates the power trace of the system [3]. Final values for power, total power, reactivity, total yield are given in a text output file. Each flux shape calculation is provided along with other problem specific files in a final output directory.

TDKENO has a wide array of parameters that can be turned on or off depending on the problem. Details of these are provided in Ref [3]. We are interested in how TDKENO uses KENO so we will continue by focusing on how KENO calculates effective multiplication values and the flux shapes.

\subsection{DESCRIPTION OF KENO-VI}

The Monte Carlo code KENO-VI is primarily used for calculating effective multiplication factor and flux shape distributions. It has the ability to operate in two cross section modes, continuous and multigroup [5,6]. At present TDKENO only operates in multigroup.

KENO V.a is geometrically limited using only basic shapes such as spheres, cylinders, and cuboids for designing a core. These shapes describe a core via combinatorial geometry. The advantage is all regions are defined and determining neutron locations is computed quickly [7]. Thus KENO V.a is approximately four times faster than its counterpart KENO-VI. The reason for KENO-VI's performance is due to its ability to handle generalized geometry by describing shapes with quadratic functions [6]. This is primarily because locating neutrons within a core is time consuming when testing many quadratic functions. The advantage is cores may be modeled with less regions leading to simpler inputs and more representative models of core designs. 
Assuming there is no external source, the time-dependent Boltzmann transport equation can be converted to a stationary pseudo-critical equation, described in Equations (2.3) and (2.4), by introducing the multiplication factor, $k$, which scales the fission source to exactly balance the loss rate [5].

$$
\widehat{\Omega} \bullet \nabla \Phi(\widehat{X}, E, \hat{\Omega})+\Sigma_{t}(\widehat{X}, E) \Phi(\widehat{X}, E, \widehat{\Omega})=q(\widehat{X}, E, \widehat{\Omega})
$$

Equation 2.3 is an eigenvalue problem with the total source defined as

$$
q(\widehat{X}, E, \widehat{\Omega})=\iint d E^{\prime} d \Omega^{\prime} \Phi\left(\widehat{X}, E^{\prime}, \widehat{\Omega}\right) \Sigma_{s}\left(\widehat{X}, E^{\prime} \rightarrow E, \widehat{\Omega^{\prime}} \rightarrow \widehat{\Omega}\right)+\frac{1}{k} Q(\widehat{X}, E, \widehat{\Omega}),
$$

where:

$k=k_{\text {eff }}=$ is the largest eigenvalue of the homogeneous equation, $Q(\widehat{X}, E, \widehat{\Omega})=$ is

the fission source at position $\widehat{X}$ for energy $E$ and direction $\widehat{\Omega}$ (all fission contributions to point $E$ from all energy points in the previous generation). Eigenvalue problems lend themselves to being solved computationally so this form is preferential for determining $k_{\text {eff. }}$.

Equation 2.3 is further modified for the continuous and multi-group methods. The result of applying each respective method is described in Ref. [5]. While each yields a different expression, the Monte Carlo approach to solving each equation is similar. In this form solving the eigenvalue equation yields the effective multiplication factor. Since KENO is often used for criticality calculations this form is useful as it gives an idea about how the neutron population is behaving.

TDKENO finds the flux at a particular time by converting the time dependent transport equation into a stationary one. The conversion of the time derivative term is accomplished by approximating it with the backward difference approach [3]. The equation instead has a fixed source and the total cross section is scaled by the term $1 / v\left(t_{n+t}-t_{n}\right)$, where $v$ is velocity and $n$ represents the time at which the shape calculation is performed. A complete description of the equation 
can be found in Ref. [5]. Solving the stationary neutron transport equation for the flux is done by applying the normalization condition.

As previously noted KENO V.a only supports basic shapes such as spheres, cylinder, squares, are available for designing a core [7]. It has other restrictions such as regions may not intersect, or be rotated. To better capture the geometry of cores such as TREAT, KENO-VI has been modified to perform the role of KENO V.a. KENO-VI supports a wide array of geometries including planes, dodecahedrons, wedges, parallelepiped, etc.

\subsection{KENO-VI INTEGRATION WITH TDKENO}

KENO-VI has gone through several iterations as SCALE has been developed. We incorporated and modified KENO-VI code from SCALE 6.1. Flux shapes are printed to an output file each time they are calculated. To save time, future iterations of TDKENO will write files to binary and then have a converter to view when needed. The user dictates how many flux shapes are desired based on the problem. The optimal number of flux shapes for a given problem is of interest as it is computationally expensive. Modifying KENO-VI files for TDKENO required updating the build environment for TDKENO. Currently, CMAKE is used to handle the building of TDKENO, which has hundreds of files across many directories. As of now CMAKE scripts have been written so TDKENO can be built on Linux and Mac OSX based computers.

\subsection{APPLICATION TO TREAT (MEASURED TRANSIENT DATA)}

The ultimate goal of modifying and verifying the updates to TDKENO is to model the transient behavior of the Transient Reactor Test Facility (TREAT) at Idaho National Laboratory (INL). The TREAT reactor at INL was originally designed to generate a short high-energy neutron pulse leading to rapid energy deposition within mockups of fast reactor fuel elements under controlled conditions 
without harm to the reactor itself [8]. The fuel elements are composed of highly enriched (93.1\%) $\mathrm{UO}_{2}$ dispersed homogenously within a graphite mixture with a graphite to uranium ratio of $10,000: 1$. The fuel elements are $10.0584 \mathrm{~cm}$ square with $1.5875 \mathrm{~cm}$ chamfered corners for air coolant flow. The active fuel length is 122.2375 $\mathrm{cm}$ and the total fuel element length is $247.65 \mathrm{~cm}$.

The fuel is encased in a Zircalloy-3 can and capped with graphite reflectors on the top and bottom encased in a 6063-aluminum can. Control elements are identical in size and design to the fuel elements except they contain holes to allow insertion and removal of control rods. The control rods consist of a $4.445 \mathrm{~cm}$ outer radius Zircaloy-2 tube that contains the carbon steel tube packed with $\mathrm{B}_{4} \mathrm{C}$ powder, with a pure carbon follower. There are a total of 20 control elements with $152.4 \mathrm{~cm}$ active absorber length. These control elements are grouped into three banks: 4 Compensation rods, 8 Control/Shutdown rods, 8 Transient rods; within each bank rods are paired and driven by a single motor. During transient operation, the Control/Shutdown rods are used at the end of the transient to force the reactor into a subcritical state. The Compensation rods are used to maintain the reactivity of the core during transient operation. The Transient rods are the rods that are quickly driven on a high pressure hydraulic system to initiate a transient.

During transient operation, forced air flow is typically disengaged (it is used primarily for post-transient cooling). Thus the graphite acts as a giant heat sink for the $\sim 20$ micron fuel particles with time lags (energy transfer into graphite) on the order of milliseconds. In a temperature-limited transient, the increased graphite temperature shifts the thermal Maxwellian neutron population to a higher average energy, generating a negative reactivity feedback which inevitably offsets the initial reactivity insertion, returning the reactor to steady state.

TREAT is currently being reactivated after 20 years in a fueled standby state to conduct new transient irradiation experiments. One of the key missions of the 
restarted facility will be to assess the performance of Accident Tolerant Fuel (ATF) concepts under transient Light Water Reactor (LWR) conditions.

In the past, total core power and energy release over a transient were assumed to be known only within $\pm 10 \%$, and several time-consuming low and full power transient tests were needed to calibrate energy deposition calculations prior to an experiment series. Hence there is a need to better characterize the time-dependent behavior of the TREAT core under transient conditions. This will improve operational efficiency, reduce costs, and improve pre-transient experiment predictions.

\subsection{VALIDATION and VERIFICATION of TDKENO with KENO-VI}

To validate the changes made to KENO-VI several inputs were converted directly from KENO V.a to KENO-VI and run with TDKENO. Inputs were converted with a utility provided in SCALE. We chose to use the very simple computational benchmark 16a1, an arbitrarily simplified TREAT model, and the temperaturelimited transient 2855 without feedback model of TREAT. Each input was run with the ENDF/B-VII.0 cross-section libraries. Additionally, effective multiplication factors ( $k$ eff values) and reactivity insertion $(\Delta k / k)$ were compared for transient 2855. Using the same model of transient 2855, tailored to their respective geometry packages, the reactivity insertion value was obtained using SCALE's Criticality Safety Analysis Sequence (CSAS) CSAS5 (utilizes KENO V.a) and CSAS6 (utilizes KENO-VI) respectively [6,7]. The calculated values were then compared to the measured value for validation. The motivation for such simulations is two fold. One is to ensure both KENO V.a and KENO-VI calculate the same values and any deviations between KENO V.a and KENO-VI in TDKENO may be attributed to geometrical differences. The second is to validate the behavior KENO is predicting with measured data from the M8CAL tests performed in TREAT.

\subsubsection{6-A1 BENCHMARK}


The 16-A1 benchmark problem [1] describes a one dimensional transport model of a liquid metal fast breeder reactor. This problem is one-dimensional (simulates three dimensions by utilizing reflective boundary conditions in $\mathrm{Y}$ and $\mathrm{Z}$ ), with the input details listed in Table 1.

Problems were run on a single AMD Opteron(tm) Processor 6378 at $2400 \mathrm{MHz}$ of the University of Florida's HiperGator research cluster. At this time TDKENO operates completely in serial. Future work may involve parallelizing aspects of TDKENO to take advantage of modern multi-core architecture.

For a preliminary comparison, the calculated power using TDKENO with KENO V.a and KENO-VI geometry respectively, are plotted in Fig. 2.1. Inspecting Fig. 2.1 we see excellent agreement between the power output calculated using KENOVI and KENO V.a. With the additional particles run with the KENO-VI input we see run times increase by a factor of about eight compared to KENO V.a inputs. We suspect that with additional particles near perfect agreement would be found between the two. A log time scale is used to highlight the short term detail, as the problem is on a short time scale.

Table 2.1. Initial parameters provided for the $16 \mathrm{a} 1$ inputs. Additional particles were run with KENO-VI to account for more complex geometry definitions.

\begin{tabular}{|l|l|l|}
\hline & KENO V.a & KENO-VI \\
\hline Number of generations & 1500 & 1500 \\
\hline Particles per generation & 10000 & 20000 \\
\hline Number of regions & 21 & 21 \\
\hline Number of materials & 5 & 5 \\
\hline Flux shapes & 5 & 5 \\
\hline Run time & $85 \mathrm{~min}$ & $900 \mathrm{~min}$ \\
\hline
\end{tabular}




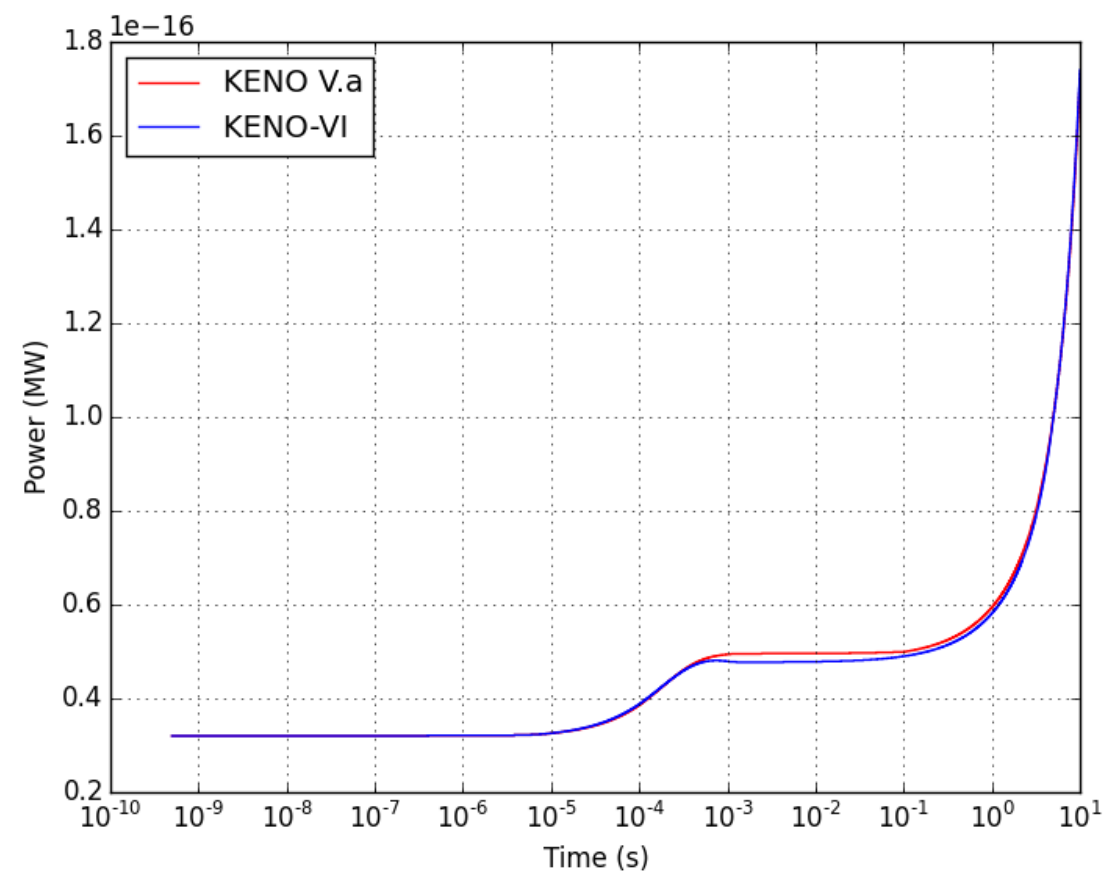

Fig. 2.1. Power comparison for $16 \mathrm{a} 1$ benchmark transient data.

Table 2.2. Input parameters for simplified TREAT.

\begin{tabular}{|l|l|l|}
\hline & KENO V.a & KENO-VI \\
\hline Number of generations & 500 & 500 \\
\hline Particles per generation & 1200 & 4000 \\
\hline Number of skipped generations & 100 & 100 \\
\hline Number of regions & 44 & 40 \\
\hline Number of materials & 7 & 7 \\
\hline Flux shapes & 3 & 3 \\
\hline Run times & 30 min & 145 min \\
\hline
\end{tabular}




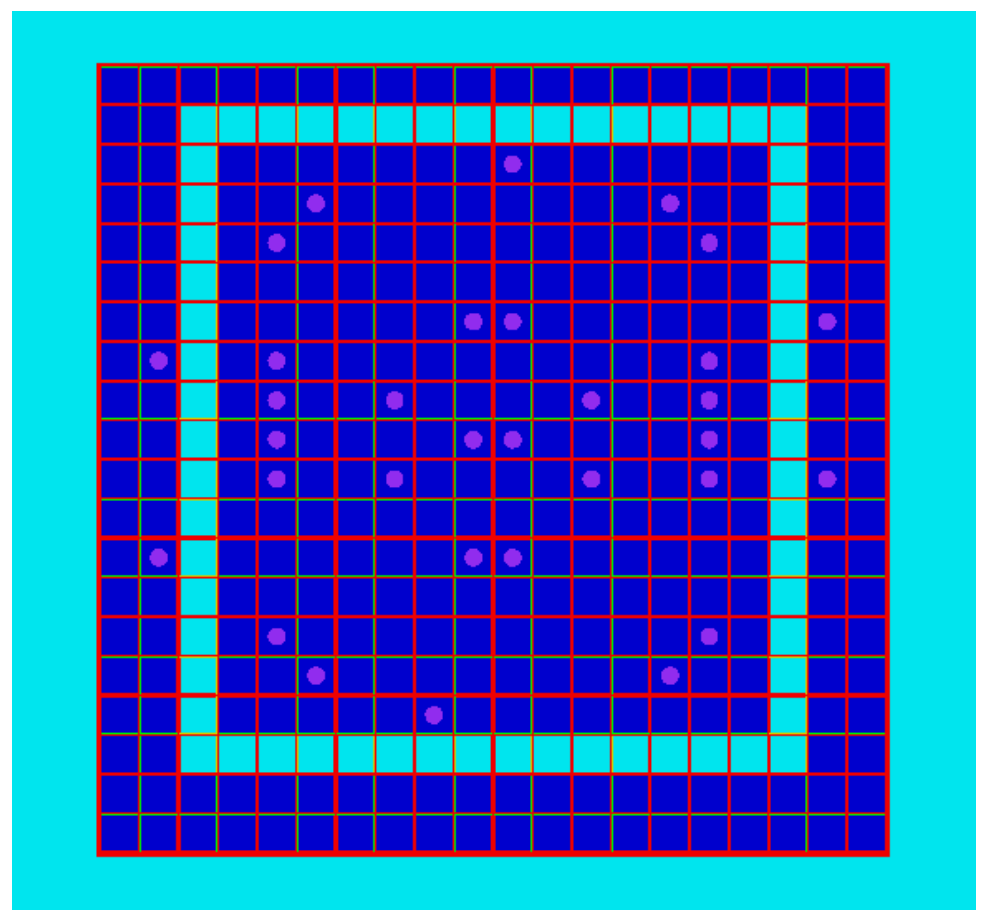

Fig. 2.2. Overhead view of the simplified TREAT core. There are far fewer regions and material types in this core. However the approximate core design, i.e placement of control rods, total size are similar to the transient 2855 model.

\subsubsection{ARBITRARILY SIMPLIFIED TREAT (Single Element)}

As a precursor to simulating the full transient 2855 a simplified version of TREAT was first modeled. A visual representation of the core can be seen in Fig. 2.2. We simulated rod withdrawal at time zero followed by feedback at 1.2, 1.3 and 1.4 seconds with Doppler-broadened cross sections at $307 \mathrm{~K}, 323 \mathrm{~K}$ and $348 \mathrm{~K}$. Other problem specifics are given in Table 2.2. Once again the calculated power plotted as a function of time in Fig. 2.3. Only minor deviations such as the power peaking sooner for transient in the KENO-VI case are evident in Fig. 2.2. Running the KENO-VI inputs with additional particles aided in the convergence of the calculated power between KENO V.a and KENO-VI. 


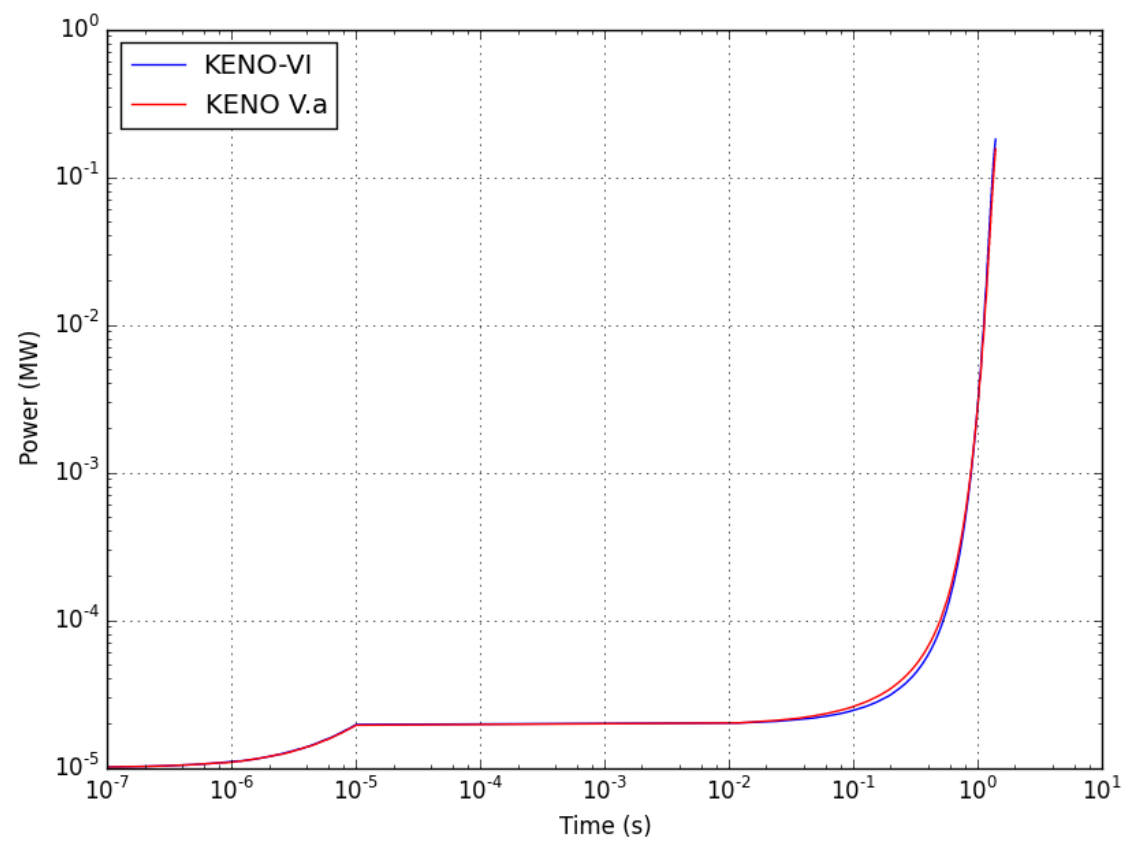

Fig. 2.3. The calculated power for the arbitrarily simplified model of TREAT is presented on a log-log scale. Agreement between KENO V.a and KENO-VI appears excellent.

\subsubsection{TEMPERATURE-LIMITED 2855 TRANSIENT}

The final goal of modifying TDKENO is to better model the transient behavior of the M8CAL tests performed at INL identified as 2855, 2856, 2857 [9]. Transient calculations were performed using both KENO inputs. To simulate control rod removal, the same input was varied for the adjoint, pre-, and post-transient configurations [8]. By this we mean the input geometry is the same but the material inside is varied, giving the appearance that the rods moved inside of the system. An overhead view of the TREAT geometry model is given in Fig. 2.4. The 2855 transient has been analyzed with TDKENO utilizing inputs from both versions of KENO and compared with reported values. Problem details are given in Table 2.4. In this case KENO-VI has more regions due to the direct conversion from KENO V.a. Future inputs of TREAT with KENO-VI inputs should be less by a factor of about three due to the elimination of the chamfered corner approximation, which is highlighted in Fig. 2.5. 


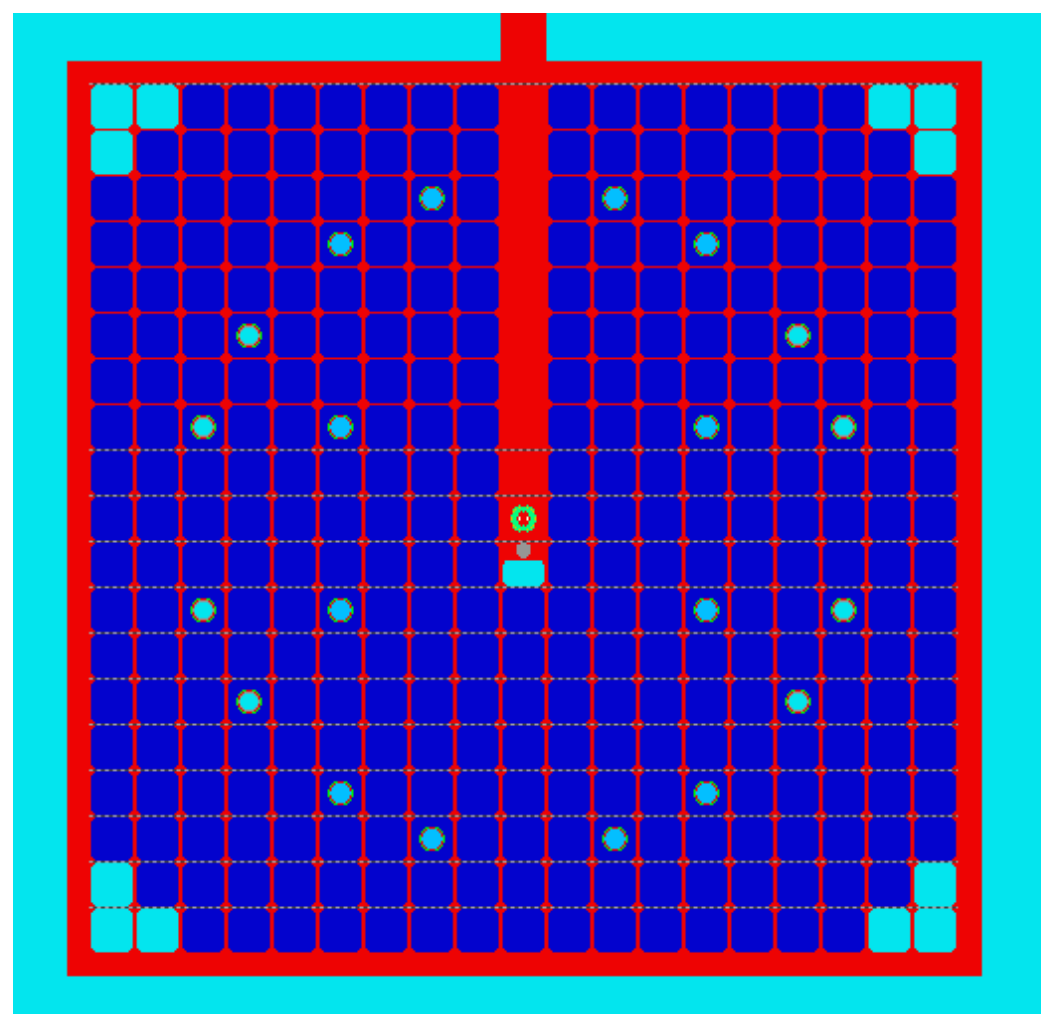

Fig. 2.4. An overhead view of the TREAT core for transient 2855.

Table 2.3. KENO V.a simulation of TREAT core in configuration for transient 2855 utilizing 2500 generations with 2000 neutrons per generation, including reported experimental values for reference [10].

\begin{tabular}{|c|c|c|c|c|c|}
\hline $\begin{array}{c}\text { SCALE } \\
\text { Library }\end{array}$ & $\begin{array}{c}\text { KENO } \\
\text { VERSION }\end{array}$ & Adjoint & Pre-Transient & Post-Transient & $\Delta \boldsymbol{k} / \boldsymbol{k}$ \\
\hline Reported & N/A & N/A & N/A & N/A & $1.80 \%$ \\
\hline CE-v7-238 & V.a & N/A & $1.0096 \pm 0.0003$ & $1.02896 \pm$ & $1.92 \%$ \\
\hline v7-238 & V.a & $1.0084 \pm$ & $1.01019 \pm$ & $1.03007 \pm$ & $1.97 \%$ \\
& & 0.0044 & 0.00024 & 0.00024 & \\
\hline CE-v7-238 & VI & N/A & $0.99563 \pm$ & $1.01438 \pm$ & $1.88 \%$ \\
\hline v7-238 & VI & $0.995 \pm$ & $0.99819 \pm$ & $1.01742 \pm$ & $1.92 \%$ \\
\hline
\end{tabular}



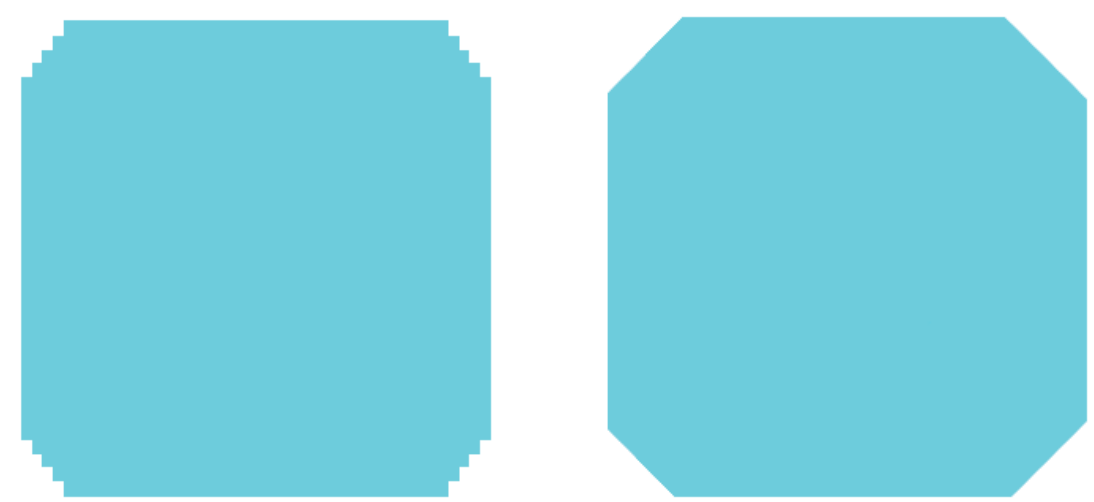

Fig. 2.5. On the left is an overhead view of a typical rod modeled with KENO V.a. The chamfered corners are approximated with a series of cuboids. In KENO-VI the approximation may be replaced with parallel planes. This leads to more accurate simulation of the core and inputs with far fewer regions.

Table 2.4. Input parameters for transient 2855 runs with TDKENO. Additional regions are in the KENO-VI input because it was directly converted from KENO V.a inputs. For unspecified and voided regions KENO-VI requires specification, which lead to the creations of additional regions.

\begin{tabular}{|l|l|l|}
\hline & KENO V.a & KENO-VI \\
\hline Number of generations & 1200 & 1200 \\
\hline Particles per generation & 2500 & 2500 \\
\hline Number of skipped generations & 10 & 10 \\
\hline Number of regions & 1771 & 1813 \\
\hline Number of materials & 21 & 21 \\
\hline Run Time & 16 hours & 55 hours \\
\hline
\end{tabular}

To obtain a preliminary idea of the reactivity calculated by TDKENO, the KENO V.a inputs with CSAS in the adjoint, pre, and post transient configurations were run to calculate reactivity insertion $\Delta k / k$. In the M8CAL test this was found to be $1.80 \%$. Table 2.3 provides the calculated reactivity insertions values.

Agreement between the two KENO option results is seen but there is clear 
deviation from the reported value.

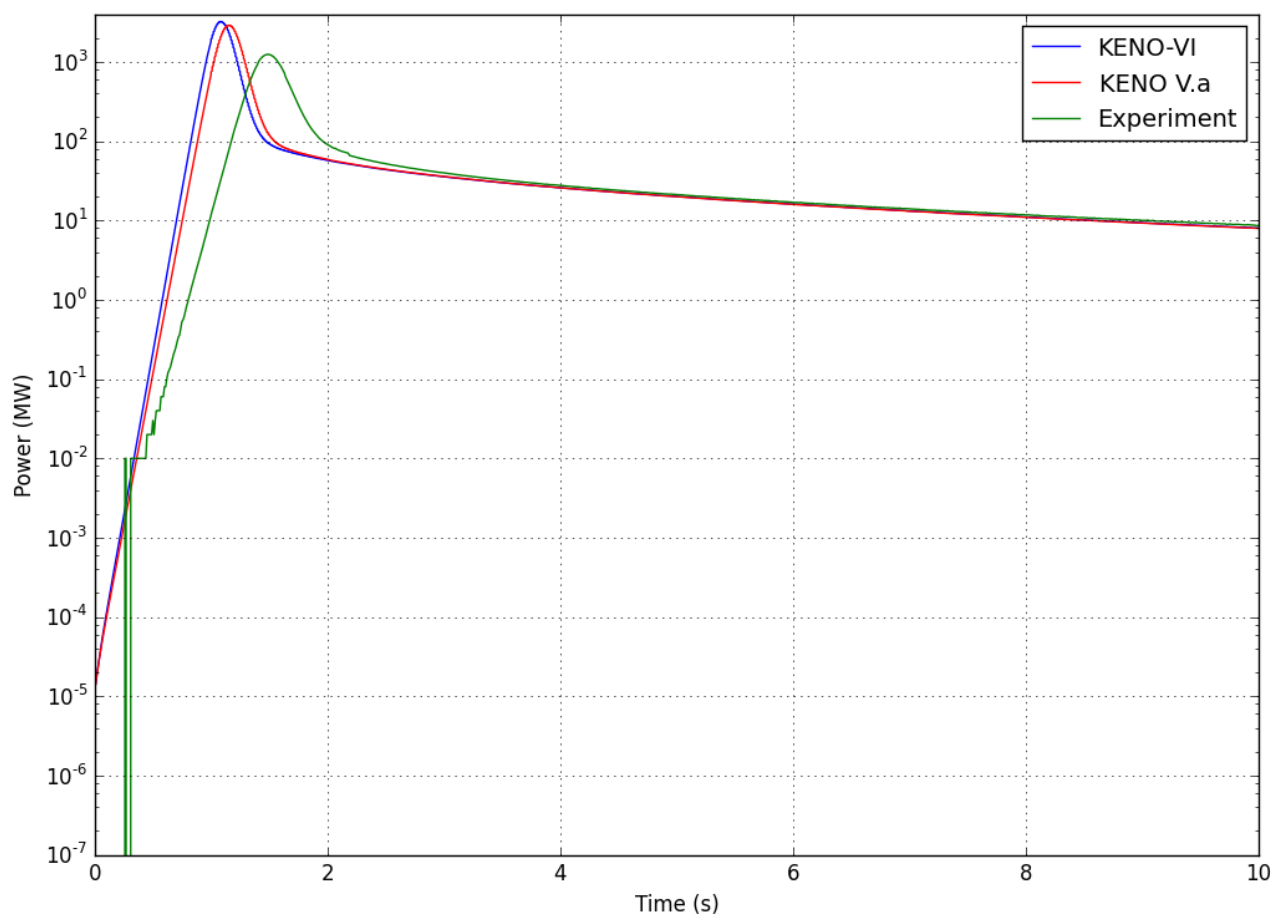

Fig. 2.5. Calculated power is compared between KENO V.a and KENO-VI. The experimental data is plotted to validate TDKENO. Agreement between KENO V.a and KENO-VI is satisfactory. However, with both codes TDKENO still calculated the peak power to be too high and early compared with M8 calibration tests.

To compare the calculated power with TDKENO using both versions of KENO with experiment we plot each in Fig. 2.6. We are interested in the main power spike occurring in the first second. Therefore, the results have only been plotted up to 10 seconds. The power scale is logarithmic to capture the details of the power spike at early time steps. This time period is important because the rods were removed at $t=0$, traveling a distance of 13.6 in. over $0.13 \mathrm{~s}$. Experimental data appears blocky at first. We suspect this was due to the limit of the recording instrument. After about 0.5 seconds the experimental data smooths out and follows the same behavior as calculated in TDKENO. With each KENO input the calculated power appears to spike earlier and with a larger amplitude than 
experiment. We suspect the disagreement appears because the KENO inputs calculate a $\Delta k / k$ value larger than $1.8 \%$, which may indicate the reported rod positions before the transient are not accurate.

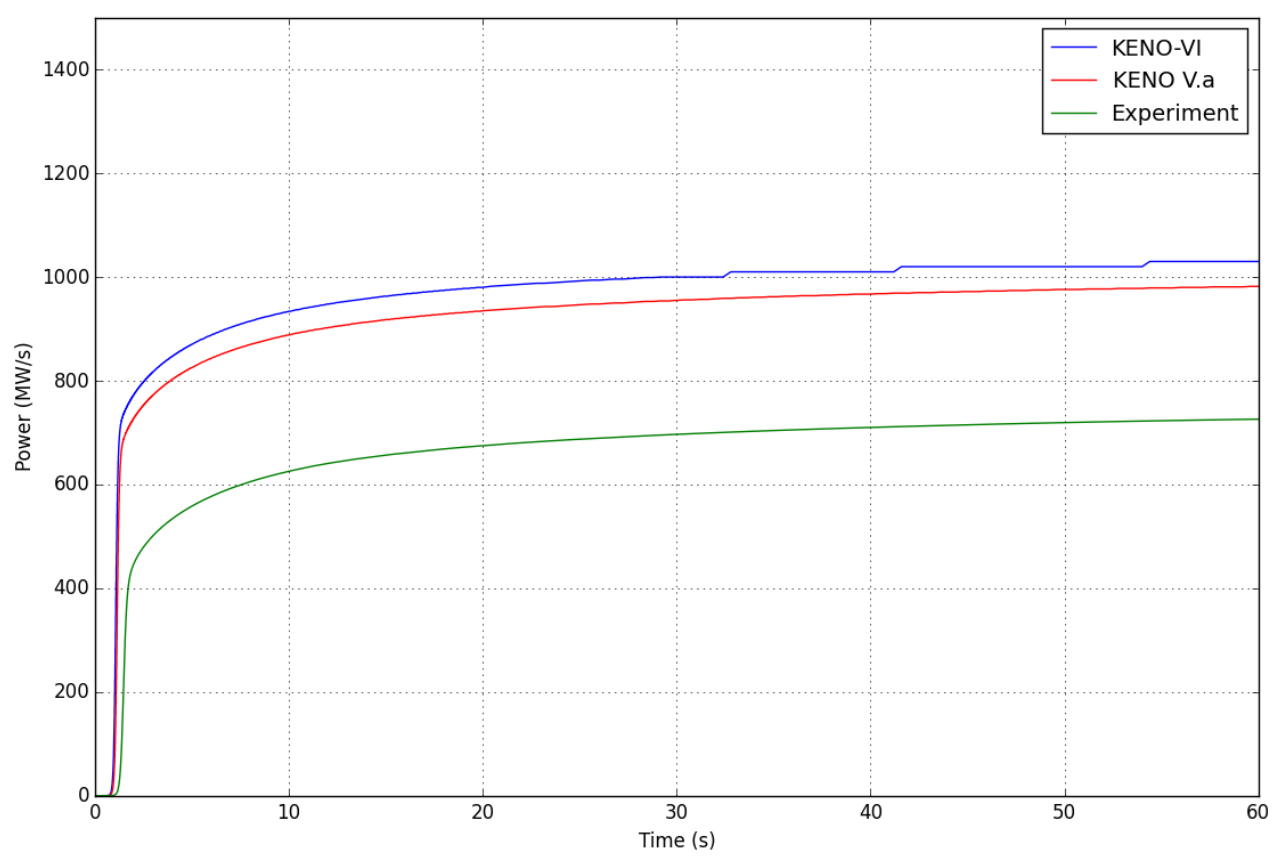

Fig. 2.6. The total yield calculated with TDKENO using both KENO inputs compared with data from M8CAL test. Clear deviation from the experiment is evident with both KENOs. We suspect this is due to inconsistency with the data reported in the M8CAL tests and the difference in $\Delta \boldsymbol{k} / \boldsymbol{k}$ values between TDKENO and experiment [10].

Analyzing Fig. 2.7 we see discrepancy between values calculated in TDKENO and the experiment. Here the yield, or total power is plotted against time. Again deviations from experiment are most likely due to the $\Delta k / k$ disagreement seen between TDKENO and the experimentally reported value. Furthermore there appears to be disagreement on the total yield between the data provided in the M8CAL for plotting and what was reported as the maximum yield. For transient 2855 it was reported that $792 \pm 79.2 \mathrm{MJ}$ were deposited in the core over a 60 second period [10]. If indeed that is the true yield experimentally found then the 
yield found with TDKENO appear to be within two standard deviations of the experiment. Moving forward we will ascertain which is the true yield, the data from the plot or the final value given in the M8CAL report.

The results TDKENO is calculating in this case are not as close as we hoped. However, we are confident in TDKENO's ability to describe transient behavior based on the preliminary results from running TDKENO with available KENO V.a inputs for transient cases 2856, and 2857 from the M8CAL tests. Work on performing full analyses with KENO-VI inputs underway.

Run times for TDKENO are quite long for inputs such as transient 2855. The number of regions is high with both KENO V.a and KENO-VI inputs so flux shape calculations take significant amounts of time. To see if it were possible to use less flux shapes and still achieve good results we took the fluxes calculated from a region containing all of the control rods, and moderators. Then we plotted each flux shape on top of each other as in Fig. 2.8. The first 4-5 flux shapes vary but after that they are all approximately the same. Fig. 2.9 highlights the fluxes at low-energy groups (200-238) and the convergence of fluxes becomes apparent. Looking back at the plots of power and yield for this transient it is reasonable to expect the fluxes do not change after a few shape calculations. The rods are removed quickly from the core in the first second leading to the spike in power. After the spike the power and yield approach a steady value thus leading to relatively little change in the flux shapes. We suspect that fewer shape calculations after about 2 seconds would yield the same results and improve the calculation time for this transient.

\subsection{FUTURE WORK}

The next step is to analyze transients 2856 , and 2857 with TDKENO using both KENO inputs. Additionally, we are working to create a KENO-VI input that utilizes planes for the chamfered corners. The number of regions should decrease by a factor of 3 , which will lead to significant reduction in computational time. 
To further improve computational time we are looking to employ KENO and other modules from SCALE 6.2 once a more stable beta is released. The advantage of SCALE 6.2 is its parallel KENO capabilities. A final goal will be to parallelize method inside of TDKENO well. OpenMPI provides many built in Fortran parallelization subroutines that would lend itself to the calculations we would like to parallelize.

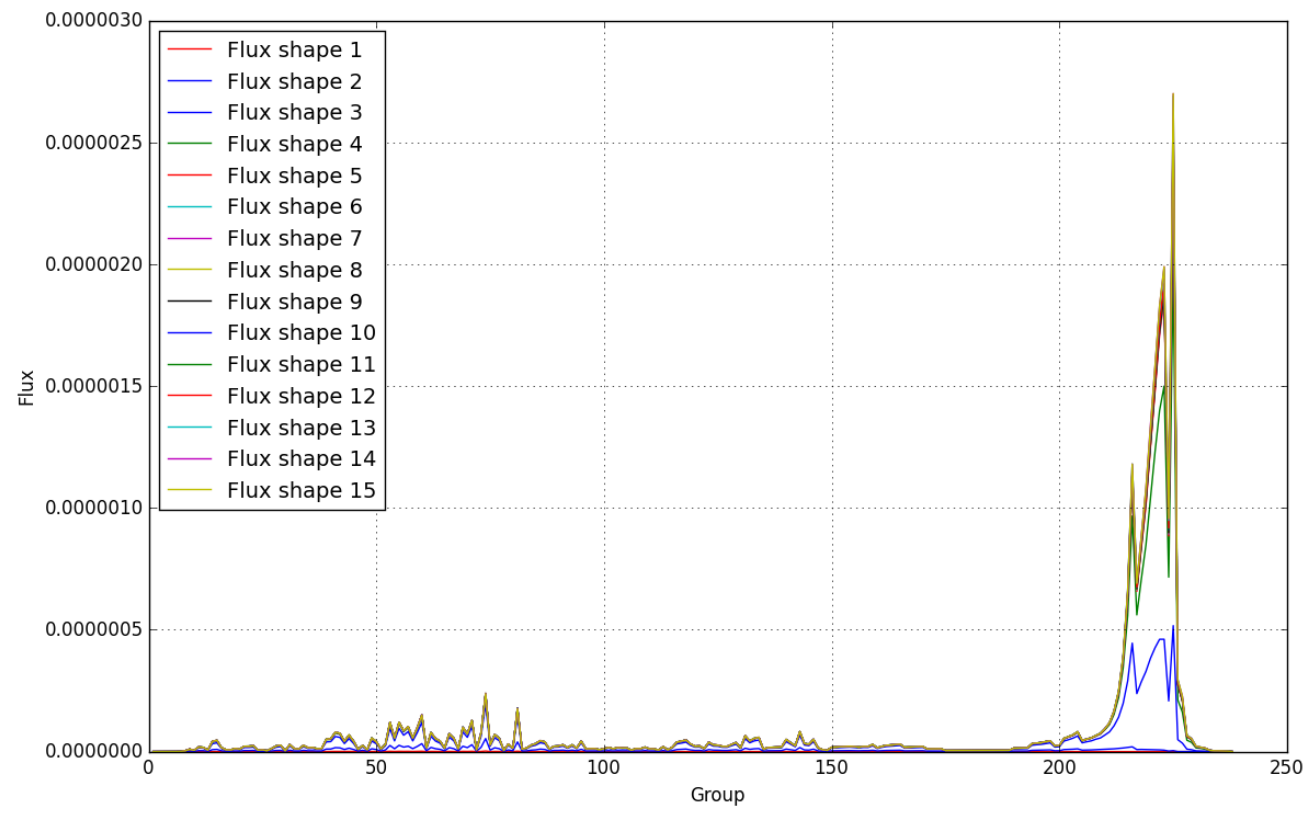

Fig. 2.7. The calculated fluxes from KENO-VI for the first 15 shape calculations are plotted as a function of group. In this multigroup case we had 238 groups. The final two shapes are not apparent on this scale and were omitted for clarity. The first 4-5 shapes differ significantly and then all approach the same shape. This conclusion is easier seen by looking at the higher energy groups, where the more flux detail is evident.

\subsection{SUMMARY}

The task of modifying TDKENO to use KENO-VI for transport calculations has been completed on time. The new option has been verified against KENO V.a with a number of models. Transient 2855 has been tested with TDKENO using an input converted to KENO-VI geometry directly from a KENO V.a input. The 
next goal will be to create a simpler model that is better representative of TREAT's geometry using KENO-VI inputs. Comparison of TDKENO's calculated power and the measured data from M8CAL tests appear to diverge in a few points. The experimental data peaks later and with less power than is calculated with TDKENO. We suspect this is due to the disagreement in reported versus the data we were given for transient 2855.

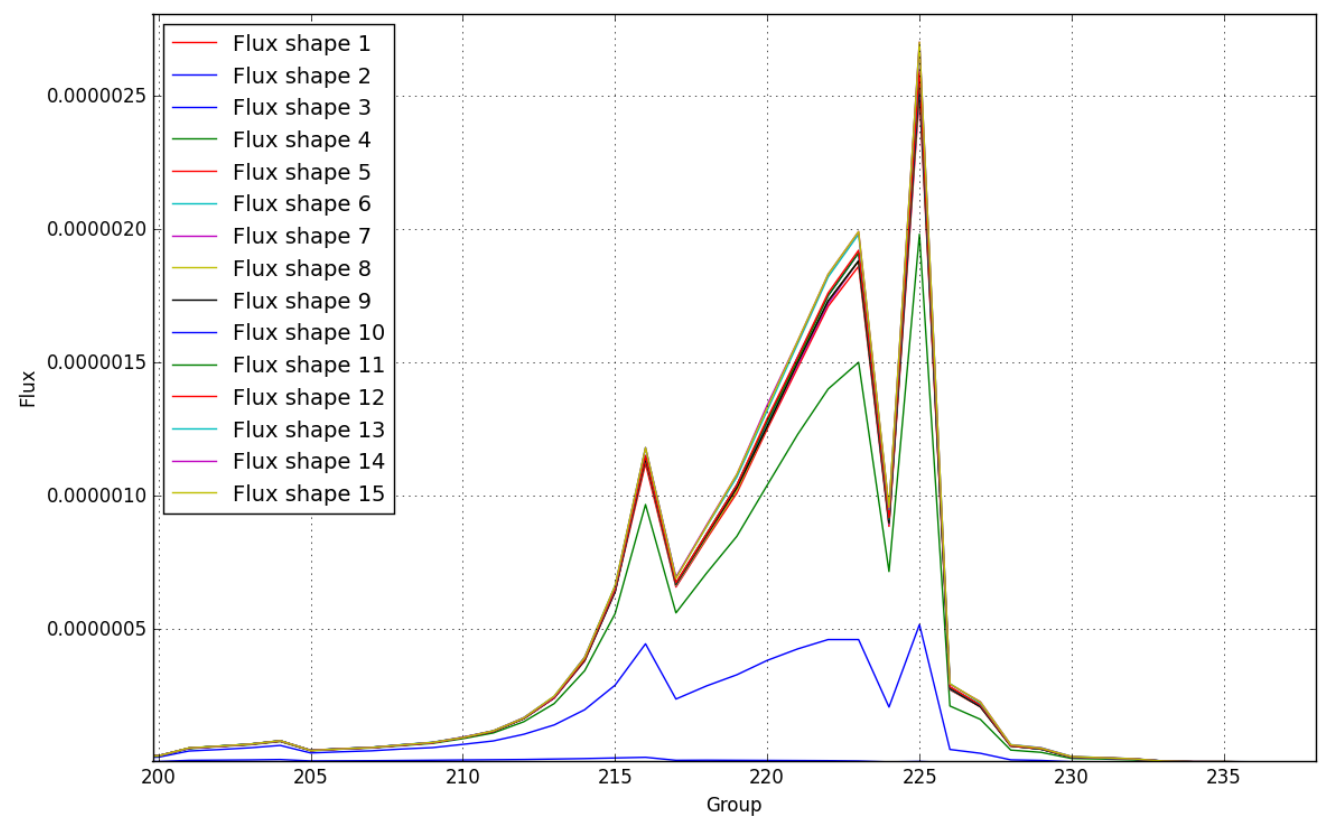

Fig. 2.8. Here we examine the high energy groups from Fig. 2.8 in order to see the convergence of flux shapes as time increases. After about the $5^{\text {th }}$ flux shapes, additional ones converge to almost the same values 


\subsection{Implementation of the Improved Quasi-Static Method in Rattlesnake/ MOOSE for Time-dependent Radiation Transport Approximations}

The anticipated restart of Transient Reactor Testing (TREAT) Facility at Idaho National Laboratory (INL) has brought significant attention and opportunity to transient modeling. TREAT, which was operational from 1954 to 1994, was designed to test nuclear fuels by subjecting them to various degrees of neutron pulses, from minor transients to accident cases. Neutron transient modeling has always been computationally expensive due to implicit time-stepping caused by the neutron velocity values. Even with the vast improvements in computing technology, straightforward discretization of neutron conservation equations remain computationally challenging for real-world cases. Therefore, methods that improve on computational speed significantly, with minimal detriment to accuracy, are highly desired. The Department of Energy (DOE) and INL have invested a substantial effort in Modeling and Simulation (M\&S) for TREAT. This report presents an implementation of the improved quasi-static (IQS) method for time-dependent neutron transport and diffusion equations within the multiphysics framework MOOSE [11], notably its radiation transport application, RATTLESNAKE.

The improved quasi-static (IQS) method is a spatial kinetics method that involves factorizing the flux solution into space- and time-dependent components $[12,13]$. These components are the flux amplitude and its shape. Amplitude is only timedependent, while the shape is both space- and time-dependent. However, the impetus of the method is the assumption that the shape is only weakly dependent on time; therefore, the shape may not require an update at the same frequency of the amplitude function, but only on macro-time steps. As opposed to other forms of quasi-static methods, the IQS method is not an approximation; the shape is updated consistently. The results of IQS may only differ from straightforward, temporal discretization because the time discretization truncation error in the shape increases with a larger time-step size. 
Implementing IQS in RATTLESNAKE (a MOOSE-based application) is an obvious endeavor to enable high-fidelity modeling of the TREAT facility. The rest of this section will briefly describe the derivation of IQS (in the diffusion setting for brevity), its current application to RATTLESNAKE using the MultiApp Picard iteration capabilities of MOOSE, and an outlook regarding ongoing and future developments.

\subsection{BACKGROUND ON THE IQS METHOD}

In this Section, we recall the equations for the IQS method, starting from the standard multigroup diffusion equations written below:

$$
\begin{gathered}
\frac{1}{v^{g}} \frac{\partial \phi^{g}}{\partial t}=\frac{\chi_{p}^{g}}{k_{e f f}} \sum_{g^{\prime}=1}^{G}(1-\beta) v^{g^{\prime}} \Sigma_{f}^{g^{\prime}} \phi^{g^{\prime}}-\left(-\nabla \cdot D^{g} \nabla+\Sigma_{r}^{g}\right) \phi^{g} \\
+\sum_{g^{\prime} \neq g}^{G} \sum_{s}^{g^{\prime} \rightarrow g} \phi^{g^{\prime}}+\sum_{i=1}^{I} \chi_{d, i}^{g} \lambda_{i} C_{i}, \quad 1 \leq g \leq G \\
\frac{d C_{i}}{d t}=\frac{\beta_{i}}{k_{e f f}} \sum_{g=1}^{G} v^{g} \sum_{f}^{g} \phi^{g}-\lambda_{i} C_{i}, \quad 1 \leq i \leq I
\end{gathered}
$$

with

$$
\beta=\sum_{i=1}^{I} \beta_{i}
$$

Factorization is an important step in the derivation of the IQS method. The factorization approach leads to a decomposition of the multigroup flux into the product of a time-dependent amplitude $(p)$ and a space-/time-dependent multigroup shape $(\varphi)$ :

$$
\phi^{g}(\vec{r}, t)=p(t) \varphi^{g}(\vec{r}, t)
$$

To obtain the amplitude equations, the multigroup equations are multiplied by a time-independent weighting function, typically the initial adjoint flux $\left(\phi^{*}\right)$, and 
then integrated over phase-space. For brevity, the inner product over space will be represented with parenthetical notation:

$$
\int_{D} \phi^{* g}(\vec{r}) f^{g}(\vec{r}) d^{3} r=\left(\phi^{* g}, f^{g}\right)
$$

Therefore, equations (3.1) and (3.2) become:

$$
\begin{gathered}
\frac{d p}{d t}\left(\phi^{* g}, \frac{1}{v^{g}} \varphi^{g}\right)+p \frac{d}{d t}\left(\phi^{* g}, \frac{1}{v^{g}} \varphi^{g}\right) \\
=p\left(\phi^{* g},\left\{\frac{\chi_{p}^{g}}{k_{e f f}} \sum_{g^{\prime}=1}^{G}(1-\beta) v^{g^{\prime}} \Sigma_{f}^{g^{\prime}} \varphi^{g^{\prime}}-\left(-\nabla \cdot D^{g} \nabla+\Sigma_{r}^{g}\right) \phi^{g}\right.\right. \\
\left.\left.+\sum_{g^{\prime} \neq g}^{G} \Sigma_{s}^{g^{\prime} \rightarrow g} \phi^{g^{\prime}}\right\}\right) \\
+\sum_{i=1}^{I} \chi_{d, i}^{g} \lambda_{i}\left(\phi^{* g}, C_{i}\right), 1 \leq g \leq G \\
\frac{d}{d t}\left(\phi^{* g}, C_{i}\right)=\frac{1}{k_{e f f}} p \sum_{g=1}^{G}\left(\phi^{* g}, \beta_{i} v^{g} \Sigma_{f}^{g} \phi^{g}\right)-\left(\phi^{*} .\right.
\end{gathered}
$$

In order to impose uniqueness of the factorization, one requires the following:

$$
\sum_{g=1}^{G}\left(\phi^{* g}, \frac{1}{v^{g}} \varphi^{g}\right)=\text { constant }
$$

After defining $\xi_{i}=\frac{\left(\phi^{*}, C_{i}\right)}{\left(\phi^{*}, \frac{1}{\nu} \varphi\right)}$, equations (3.6) and (3.7) become:

$$
\begin{aligned}
& \frac{d p}{d t} \\
& =\frac{\left(\phi^{* g},\left\{\frac{\chi_{p}^{g}}{k_{e f f}} \sum_{g^{\prime}=1}^{G}(1-\beta) v^{g^{\prime}} \Sigma_{f}^{g^{\prime}} \varphi^{g^{\prime}}-\left(-\nabla \cdot D^{g} \nabla+\Sigma_{r}^{g}\right) \phi^{g}+\sum_{g^{\prime} \neq g}^{G} \Sigma_{s}^{g^{\prime} \rightarrow g} \phi^{g^{\prime}}\right\}\right)}{\left(\phi^{* g}, \frac{1}{v^{g}} \varphi^{g}\right)} p
\end{aligned}
$$




$$
\begin{gathered}
+\sum_{i=1}^{I} \frac{\left(\phi^{* g}, \chi_{d, i}^{g} \lambda_{i} C_{i}\right)}{\left(\phi^{* g}, \chi_{d, i}^{g} C_{i}\right)} \xi_{i}, 1 \leq g \leq G \\
\frac{d \xi_{i}}{d t}=\frac{1}{k_{e f f}} p \sum_{g=1}^{G} \frac{\left(\phi^{* g}, \beta_{i} \nu^{g} \Sigma_{f}^{g} \phi^{g}\right)}{\left(\phi^{* g}, \frac{1}{v g} \varphi^{g}\right)}-\sum_{g=1}^{G} \frac{\left(\phi^{* g}, \chi_{d, i}^{g} \lambda_{i} C_{i}\right)}{\left(\phi^{* g}, \chi_{d, i}^{g} C_{i}\right)} \xi_{i} 1 \leq i \leq I
\end{gathered}
$$

It is convenient to define the effective reactivity, delayed-neutron fraction, and delayed-neutron precursor decay constant as such:

$$
\begin{gathered}
\frac{\rho}{\Lambda}=\frac{\sum_{g=1}^{G}\left(\phi^{* g},\left\{\frac{\chi_{p}^{g}}{k_{e f f}} \sum_{g^{\prime}=1}^{G} \nu^{g^{\prime}} \Sigma_{f}^{g^{\prime}} \varphi^{g^{\prime}}-\left(-\nabla \cdot D^{g} \nabla+\Sigma_{r}^{g}\right) \phi^{g}+\sum_{g^{\prime} \neq g}^{G} \Sigma_{s}^{g^{\prime} \rightarrow g} \phi^{g^{\prime}}\right\}\right)}{\sum_{g=1}^{G}\left(\phi^{* g}, \frac{1}{v g} \varphi^{g}\right)} \\
\frac{\bar{\beta}}{\Lambda}=\sum_{i=1}^{I} \frac{\bar{\beta}_{i}}{\Lambda}=\frac{1}{k_{e f f}} \frac{\sum_{i=1}^{I} \Sigma_{g=1}^{G}\left(\phi^{* g}, \beta_{i} \nu^{g} \Sigma_{f}^{g} \phi^{g}\right)}{\sum_{g=1}^{G}\left(\phi^{* g}, \frac{1}{v g} \varphi^{g}\right)} \\
\bar{\lambda}_{i}=\frac{\sum_{g=1}^{G}\left(\phi^{* g}, \chi_{d, i}^{g} \lambda_{i} C_{i}\right)}{\sum_{g=1}^{G}\left(\phi^{* g}, \chi_{d, i}^{g} C_{i}\right)}
\end{gathered}
$$

Subsequently, the standard point reactor kinetics equations (PRKE) for the amplitude solution are obtained:

$$
\begin{aligned}
\frac{d p}{d t} & =\left[\frac{\rho-\bar{\beta}}{\Lambda}\right] p+\sum_{i=1}^{I} \bar{\lambda}_{i} \xi_{i} \\
\frac{d \xi_{i}}{d t} & =\frac{\bar{\beta}_{i}}{\Lambda}-\bar{\lambda}_{i} \xi_{i} \quad 1 \leq i \leq I
\end{aligned}
$$

Finally, the shape equations are solved for the shape. The shape equations are similar to the original diffusion equations:

$$
\frac{1}{v^{g}} \frac{\partial \varphi^{g}}{\partial t}=\frac{\chi_{p}^{g}}{k_{e f f}} \sum_{g^{\prime}=1}^{G}(1-\beta) v^{g^{\prime}} \Sigma_{f}^{g^{\prime}} \varphi^{g^{\prime}}-\left(-\nabla \cdot D^{g} \nabla+\Sigma_{r}^{g}+\frac{1}{v^{g}} \frac{1}{p} \frac{d p}{d t}\right) \varphi^{g}
$$




$$
+\sum_{g^{\prime} \neq g}^{G} \Sigma_{s}^{g^{\prime} \rightarrow g} \varphi^{g^{\prime}}+\frac{1}{p} \sum_{i=1}^{I} \chi_{d, i}^{g} \lambda_{i} C_{i}, \quad 1 \leq g \leq G
$$

However, the amplitude and shape equations form a system of coupled equations: the coefficients appearing in the PRKEs depend upon the shape solution while the shape equation has a kernel dependent on amplitude and its derivative. Because solving for the shape can be expensive, especially in two or three dimensions, it is attractive to make the assumption that the shape is weakly time-dependent so the shape can be computed after a multitude of PRKE calculations, which is the root of IQS. This is depicted schematically in Fig. 3.1:

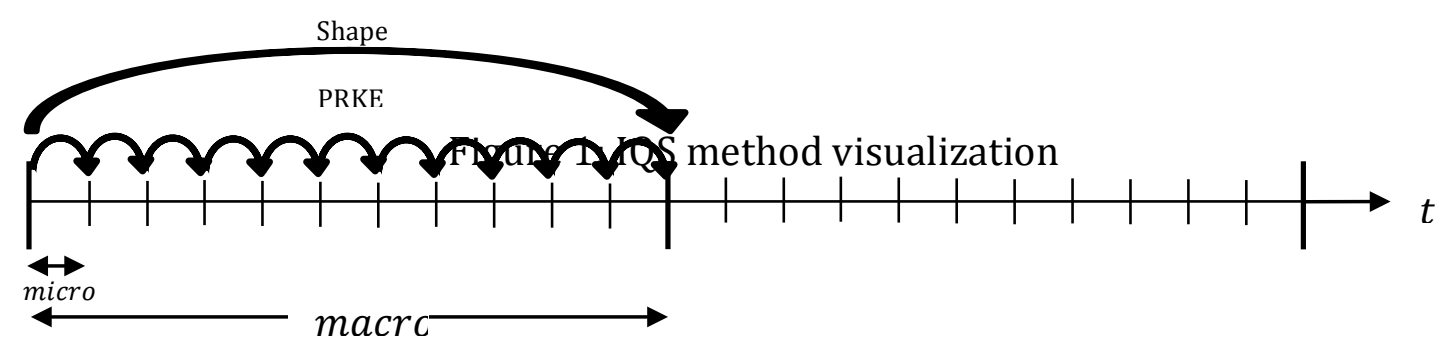

Fig. 3.1. Representation of macro-step shape calculations and micro-step amplitude calculations.

Additionally, to improve consistency and accuracy, each macro time step can be iterated so the best shape is used to compute power at the micro time steps.

Within the MOOSE framework, nonlinear systems can be tackled in two manners: with Newton's method (usually, a preconditioned Jacobian-free version) and with Picard's iterations (fixed-point method). The latter is employed in the work. This iteration process must converge the shape such that the uniqueness condition $\left(\frac{d}{d t} \sum_{g=1}^{G}\left(\phi^{* g}, \frac{1}{v^{g}} \varphi^{g}\right)=0\right)$ is preserved.

\subsection{IMPLEMENTATION IN RATTLESNAKE}


MOOSE, or Multiphysics Object-Oriented Simulation Environment, is a finiteelement-based framework developed by INL and is equipped with advanced nonlinear solvers. RATTLESNAKE is a module of MOOSE meant for neutronics and radiation transport problems. RATTLESNAKE is a radiation transport application within MOOSE and can be coupled to other physics via a Newton or a Picard approach. Implementing the IQS in RATTLESNAKE is meant to enhance its transient modeling capability. RATTLE-SNAKE utilizes an action system which initiates kernels, user objects, and post-processors; these typically need to be added manually to the input file, but due to the large phase-space of neutron transport approximations, an automated action system is invoked to add the required MOOSE objects. When implementing the IQS, the action system and its associated MOOSE objects need to be updated. For brevity, we describe the implementation in the case of the CFEM Diffusion action system; similar developments are carried out for the DFEM Diffusion action system, the $\mathrm{Sn}$ Transport action system. We discuss the CFEM Diffusion action system in detail below. Schematically, it is illustrated in Fig 3.2.

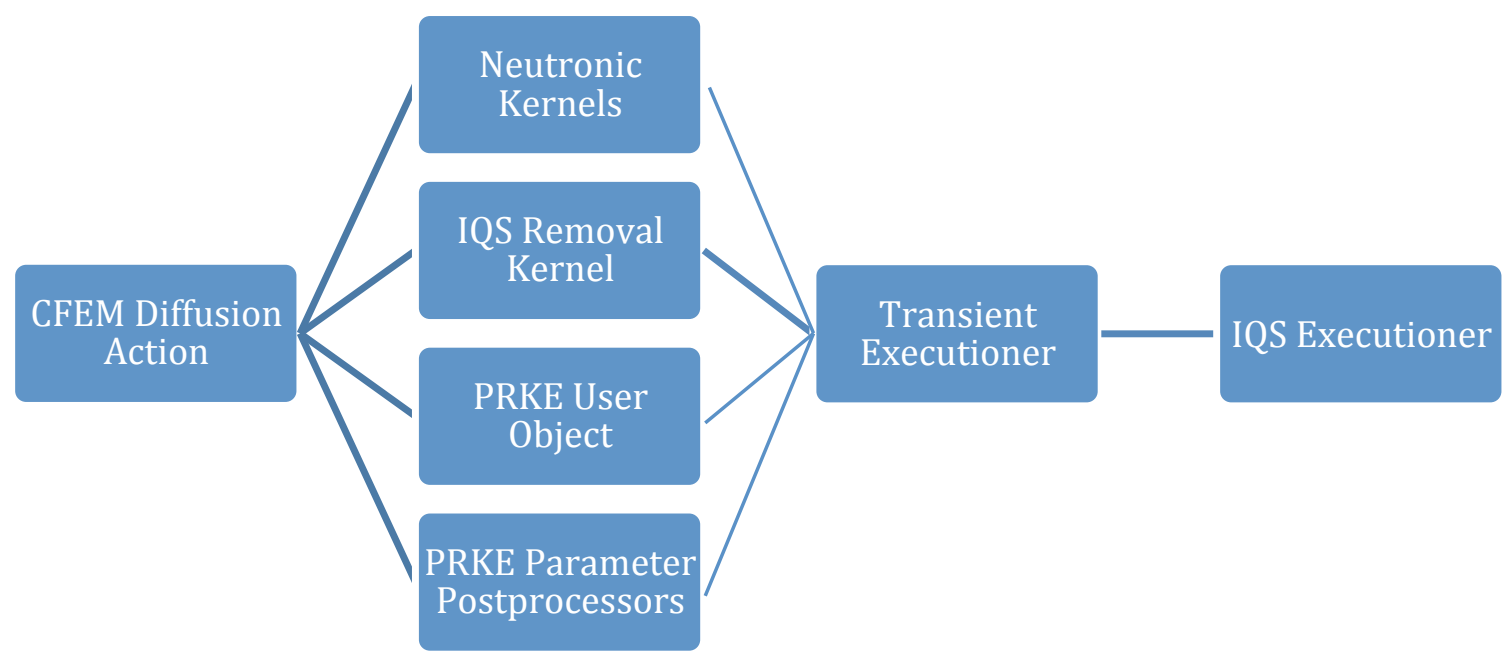

Fig. 3.2. CFEM Diffusion Action System Diagram 


\subsubsection{Action System}

IQS derives its uniqueness from the executioner type; however, some additional changes needed to be carried out in the RATTLESNAKE action system in order to support IQS execution. First, changes needed to be made in order to evaluate the shape equation. The shape equation, after some manipulation, is very similar to the time-dependent flux equation, as seen in Eq. (3.12). To enable RATTLESNAKE to solve this shape equation in lieu of the standard diffusion equation, an additional removal kernel has to be instantiated to evaluate the

quantity $\frac{1}{v p} \frac{d p}{d t} \varphi$ and added to FEM weak form when the IQS executioner is selected. Second, four postprocessors are created in order to calculate the PRKE parameters. The parameter calculations were split into the following item: $\frac{\bar{\beta}_{i}}{\Lambda}$ numerator, $\bar{\lambda}_{i}$ numerator/denominator, $\frac{\rho}{\Lambda} \frac{\bar{\beta}}{\Lambda}$ denominator, and $\frac{\rho-\bar{\beta}}{\Lambda}$ numerator. The first three are relatively simple, only relying on material properties and solution quantities. The $\frac{\rho-\bar{\beta}}{\Lambda}$ numerator requires the use of MOOSE's residual save-in feature, which saves the residual from a calculated kernel or boundary contribution in the shape evaluation to an auxiliary variable. Finally, a user object was created to pull together all the postprocessor values and carryout the numerator/denominator divisions that were then passed to the executioner.

\subsubsection{Executioner}

The IQS executioner derives from the Transient executioner in MOOSE. The IQS executioner contains a loop over micro time steps that solves the PRKEs and then passes the values for $p$ and $d p / d t$ at times corresponding to the macro-time steps into the Transient executioner in order to solve for the shape equation at each macro step. The PRKEs are solved with backward Euler within the Executioner for now but higher-order time integrators will be employed later. The IQS executioner also supplements Transient's Picard iteration process by adding its 
own error criteria for the IQS method:

$$
\operatorname{Error}_{I Q S}=\left|\frac{\left(\phi^{* g} \frac{1}{v g} \varphi^{g}\right)^{n}}{\left(\phi^{* g}, \frac{1}{v g} \varphi^{g}\right)^{0}}-1\right|
$$

The use of the Picard iteration capability of MOOSE's executioner will enable solving the nonlinear IQS equations along with other nonlinearly coupled multiphysics (e.g., thermal-hydraulics) using different time step sizes for neutronics and the other coupled physics.

\subsection{PRELIMINARY RESULTS}

This section describes results of an example that tests the IQS implementation and shows its effectiveness on computation speed and accuracy. We select a homogeneous one-group problem, subjected to a heterogeneous material change (absorption cross-section change as a ramp in time for a subset of the geometry). Fig. 3.3 shows the power at each macro time step, power is defined as the elemental integral of the flux.

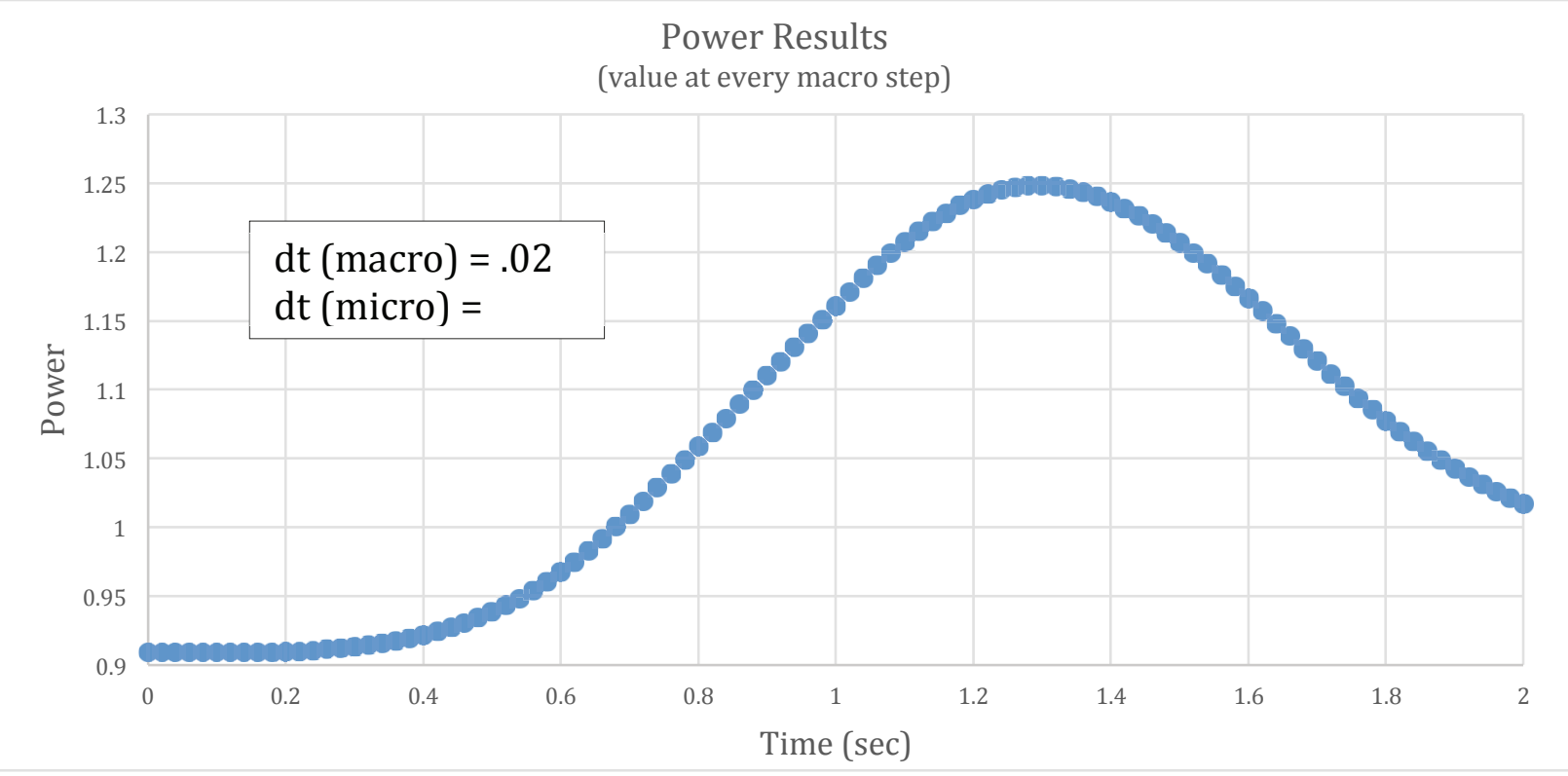

Fig. 3.3. Power level results computed as a Flux Integral Postprocessor value 


\subsection{ONGOING DEVELOPMENTS}

We have implemented the IQS method within RATTLESNAKE, part of the MOOSE framework. The implementation is complete for the CFEM Diffusion neutron equation. Ongoing developments include

1. Implementation and testing of IQS for DFEM Diffusion and Sn transport equations.

a. The application of IQS in MOOSE's Picard nonlinear solver was a relatively simple task using the object-oriented features of the framework. Once the implementation was completed for one action system (CFEM diffusion), extension to other neutron discretizations (DFEM diffusion, DFEM Sn transport) is straightforward.

2. More accurate treatment of precursors

a. Currently, precursors as treated via a first-order Backward finite difference formula with is not accurate enough for the macro time step sizes used in the IQS

b. This will be replaced with a semi-analytical treatment via an integrating factor approach with linear temporal variation of the shape over the macro-time step.

3. Verification of the IQS implementation and comparison of IQS vs. brute force temporal discretization, using

a. Manufactured solutions

b. Kinetics multi-group transient benchmarks (neutronics only, e.g., the Yasinsky 1D test cases [14], TWIGL multi-D [15], LMW multi-D [16])

c. Dynamics benchmarks (coupled neutronics theat conduction reactor dynamic problem, e.g., the LRA test case [1])

4. Implementation of a JFNK-based algorithm to resolve the nonlinearity between the amplitude/shape equations (JFNK is typically the workhorse solution technique in MOOSE). 


\subsection{IMPROVED TREATMENT OF NEUTRON PHYSICS REPRESENTATIONS WITHIN TREAT USING OPENMC}

This project seeks to develop the infrastructure for modeling of the TREAT reactor within a high fidelity computational framework. The main focus will be placed on improving MIT's OpenMC Monte Carlo code to more faithfully model the underlying physics in the TREAT reactor core, and integrating OpenMC into the MOOSE framework for accurate simulation of transients and other scenarios in which multi-physics feedback plays a major role. Coupling between OpenMC and MOOSE had already started as part of another project and this work will be leveraged in extending capabilities to the TREAT reactor. Functional expansion tallies better suited to the TREAT reactor are implemented to facilitate mapping between the constructive solid geometry model preferred by Monte Carlo code and the finite-element mesh in MOOSE.

Improving the infrastructure for modeling of the TREAT reactor will allow computational models to predict behavior of the core with sufficient accuracy as to satisfy modern regulatory concerns. Particularly, current methods do not allow easy testing of various thermal scattering models, which is an essential part of the TREAT reactor feedback mechanisms. Such studies on different graphite scattering models would require manual pre-processing of cross-section data with external codes like NJOY making the process convoluted and impractical.

The MIT OpenMC code has been moving toward on-the-fly generation of temperature dependent continuous energy cross-sections, and away from the storage of and interpolation across large tables of data. This is very conducive to conducting sensitivity studies on-the-fly, without requiring pre-processing of different data for every perturbation, and is helpful in high performance computing applications, which are often memory limited in their performance. Previous work has focused mainly on the resolved and unresolved resonance ranges, but the nature of the TREAT reactor requires further work in the thermal range. 
Section 4.1 presents the OpenMC model of the TREAT reactor. Section 4.2 provides an overview of the functional expansion tallies and their adaption for the TREAT geometry, while Section 4.4 reviews the work performed so far in incorporating a more robust thermal neutron scattering treatment in OpenMC. Concluding remarks and future work are presented in Section 4.5.

\subsection{TREAT MODEL}

In order to couple the capabilities of the OpenMC program for determining power profiles with the multi-physics capabilities of the MOOSE framework, it is necessary to have detailed models that capture the fine physical details of the system. A necessary component of this project was thus to construct an OpenMC model of the TREAT reactor. A model has been constructed that contains the basic geometry of the TREAT reactor with the associated materials [17]. Crosssectional views of a configuration of the full core along with a single fuel assembly are shown in Figs. 4.1 and 4.2.

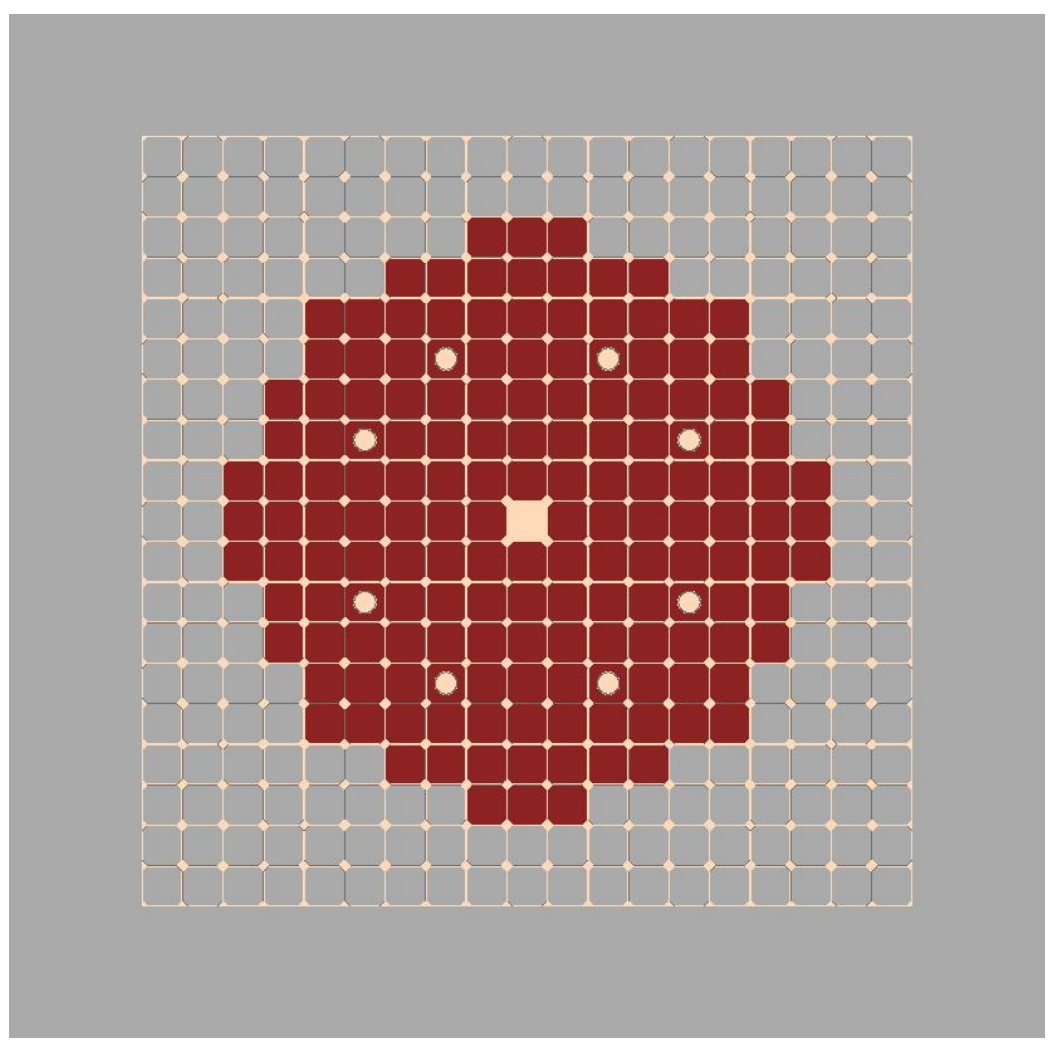


Fig. 4.1. Full core view of the TREAT OpenMC model

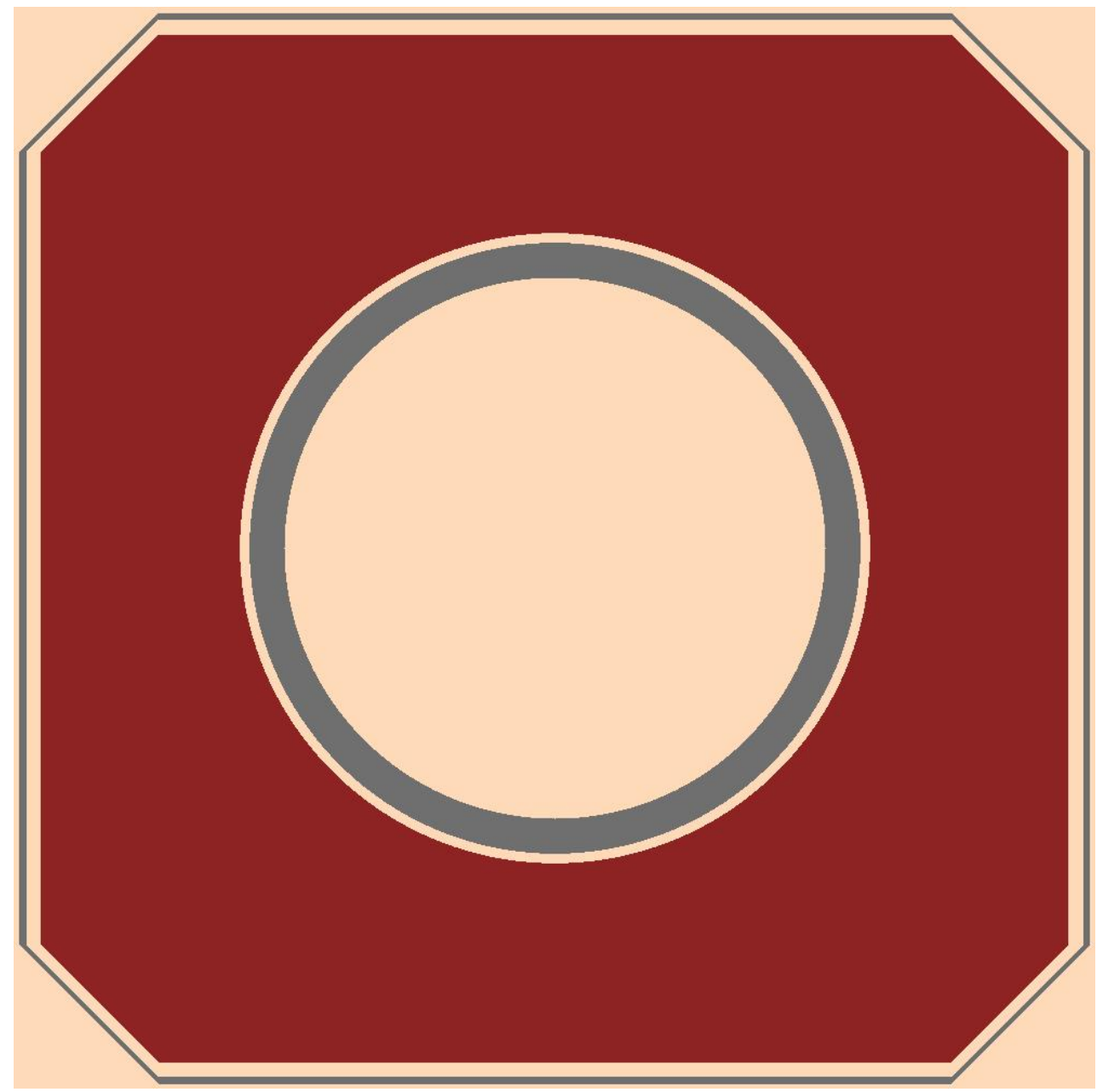

Fig. 4.2. TREAT Single Assembly OpenMC model

The model is built in a modular fashion so new loading configuration can easily and quickly be constructed. There are some features that have yet to be introduced into the model, such as control rods and fine detail on the structural materials.

\subsection{FUNCTIONAL EXPANSION TALLIES}

The fundamental obstacle to accurately transferring multi-physics data to and from a Monte Carlo simulation is the different spatial discretization schemes that are utilized in each application. If one considers a basic LWR fuel pin, the typical spatial discretization in OpenMC would consist of equal volume concentric rings 
with equally spaced azimuthal cuts. In a three-dimensional space, axial discretization would also be required along the fuel pin. For TREAT reactor applications, generating and transferring high-fidelity multi-physics data would require a similar level of geometry discretization on the nearly rectangular fuel assemblies. On these Monte Carlo meshes, quantities such as flux, fission power, and micro-rates (reaction rates divided by isotopic number density) for depletion would be tallied.

In the case of the finite element spatial discretization for the same pin or TREAT fuel element, the underlying mesh consists of an unstructured set of elements. Moreover, unlike the traditional collision-based or track-length Monte Carlo tallies, the finite element solution can consist of an arbitrary order basis set in each element which further complicates the mapping of multi-physics data between OpenMC and MOOSE.

Using functional expansion tallies (FETs) mitigates the problems related to utilizing different discretization schemes by allowing tallied quantities in OpenMC to be transferred to MOOSE applications as continuous functions. The use of continuous functions eliminates the need for interpolation schemes or tallying on an identical unstructured mesh that is computationally prohibitive.

In the initial work presented by Griesheimer et al. on the topic of FETs, Legendre polynomials were utilized in the FETs [18]. However, the derivation and implementation of FETs is nearly indifferent to the choice of basis set. For example, in the first publication related to the coupling of OpenMC and MOOSE applications, Zernike polynomials were chosen because these polynomials are defined on a unit disk which matches the LWR fuel pin geometry [19]. In the case of the TREAT reactor, Legendre polynomials will be used because Legendre Polynomials are defined on a Cartesian coordinate system which most closely matches the TREAT geometry. The functional expansion of tallied quantities is defined below: 


$$
F(x, y, z)=\sum_{l=0}^{L} \sum_{n=0}^{N} \sum_{m=0}^{M} c_{l, n, m} k_{l, n, m} P_{l}(x) P_{m}(y) P_{n}(z)
$$

In Eq. 4.1, $L, M$ and $N$ are the degrees of the Legendre expansion in the $x, y$, and $z$ directions, respectively, while $c_{l, m, n}$ is the expansion coefficient and $k_{l, n, m}$ is the normalizing coefficient. The Legendre polynomials, and expansion coefficients are defined in Eq. 4.2 and Eq. 4.3, respectively.

$$
\begin{gathered}
P_{l}(x)=\frac{1}{2^{l} l !} \frac{d^{l}}{d x^{l}}\left[\left(x^{2}-1\right)^{l}\right] \\
c_{l, n, m}=\int_{-1}^{1} d x \int_{-1}^{1} d y \int_{-1}^{1} d z \quad F(x, y, z) P_{l}(x) P_{m}(y) P_{n}(z)
\end{gathered}
$$

Using the work by Griesheimer et al. as a template, the expansion coefficients for the fission power distribution in the fuel pin can be tallied using a collision estimator with the following expression:

$$
c_{l, n, m}=\frac{1}{W} \sum_{i=1}^{N} \sum_{k=1}^{J_{i}} \frac{w_{i, k} \kappa\left(\xi_{i, k}\right) \Sigma_{f}\left(\xi_{i, k}\right)}{\Sigma_{t}\left(\xi_{i, k}\right)} P_{l}\left(\xi_{i, k}\right) P_{m}\left(\xi_{i, k}\right) P_{n}\left(\xi_{i, k}\right)
$$

where,

- $\quad W$ is the total weight of the $N$ particles

- $\quad w_{i, k}$ is the weight of particle $i$ for each collision $k$

- $\quad \xi_{i, k}$ is the phase space of particle $i$ for each collision $k$

- $\quad \kappa$ is the fission energy released

- $\quad \sum_{f}$ and $\Sigma_{t}$ are the fission and total macroscopic cross sections, respectively

- $\quad P_{l}$ is the $l^{\text {th }}$ order Legendre polynomial

From the above equation it is clear that one would need $(L+1)(M+1)(N+1)$ coefficients to do a complete three-dimensional FET over the domain. Additionally, one would also have to tally the uncertainty for each coefficient. To avoid tallying so many quantities, a 2D-1D approach is taken where the vertical 
direction, $z$, is taken to be separable from the $x$ and $y$ directions. This approach means that only $(L+1)(M+1)+(N+1)$ coefficients must be tallied. The details of this approach have been outlined by Griesheimer et al., and the 2D-1D approach has been implemented in OpenMC. If needed, further reductions are possible by eliminating the high order cross terms.

As demonstrated in the initial publication related to the coupling of OpenMC and MOOSE, FETs have been implemented in OpenMC and can be reconstructed in MOOSE applications. The initial publication, however, showed the FETs on an LWR pin cell. Currently, work is being done to determine the effects of using the FETs on an un-discretized TREAT fuel assembly. More specifically, the chamfers on the TREAT fuel assemblies and the cylindrical voids for control elements will introduce challenges when tallying quantities near this material discontinuity. For the control assemblies with a cylindrical central void, annular basis sets will be examined to mitigate the effects from the material discontinuities. Finally, it should be noted that the initial work published on the coupling of OpenMC and MOOSE applications utilized a lightweight application written specifically for proving the multiphysics transfer capabilities added to OpenMC. Current work is also focused on porting these capabilities into BISON for detailed fuel performance coupling in TREAT reactor simulations.

\subsection{THERMAL NEUTRON SCATTERING}

\subsubsection{Need for $S(\alpha, \beta)$ Update}

The TREAT reactor at Idaho National Labs is a system in which thermal neutron scattering is extremely important. This is in large part due to the prevalence of graphite and reliance on axial streaming paths. The entire core is surrounded by a thick graphite reflector, and the fuel material was historically $0.2 \%$ by weight highly enriched uranium mixed homogeneously with graphite [17]. Due to the low absorption cross section in graphite and the lattice structure of the material, 
the behavior of the thermal neutron scattering cross sections in the TREAT reactor have a very significant impact on the results observed in a simulation.

A previous study was carried out by Swanson and Harrison [20] examining the impact of assuming different lattice structures for the carbon in the TREAT fuel assemblies. Due to the proprietary nature of the fabrication methods for the TREAT fuel assemblies during much of its operation, the exact fraction of fuel that had fully annealed into the graphite structure was unknown, so many simulations had assumed perfect graphite structure. Swanson and Harrison carried out a study analyzing the difference between assuming pure graphite structure and a distribution of roughly $60 \%$ graphite, $40 \%$ free carbon to model the recently released specifications of the fuel assembly fabrication. This study found a difference in eigenvalue between the two cases on the order of $2000 \mathrm{pcm}$. Preliminary simulations of the TREAT model built within the OpenMC tool show an impact of roughly $800 \mathrm{pcm}$ from the inclusion of $S(\alpha, \beta)$ at operating temperatures compared with using a free gas model. Validation and refinement of the model is required before a definitive conclusion can be drawn, however.

Current methods for generating a thermal scattering kernel require multiple convolution integrals over large energy regimes [21]. While slow, this method is computationally simple, and does not require the use of complex numbers. Since traditional methods of cross section generation for Monte Carlo applications have involved pre-processing of nuclear data, the efficiency penalty from this methodology was insignificant. However, in moving towards on-the-fly generation of cross section values, a more efficient method must be found. Additionally, since many current Monte Carlo codes require pre-generation of cross section values with an external program, uncertainty analyses and sensitivity studies on the impact of changes in physical parameters underlying the cross sections would require a new set of tabular data to be generated for every minor perturbation and stored on the disk. For any comprehensive analysis, this is 
completely infeasible. A method of on-the-fly generation of temperature and energy dependent cross sections for all materials in a system is required.

\subsubsection{Replication of LEAPR Capabilities}

Current work has replicated the capability of nuclear data codes like NJOY to pregenerate thermal neutron scattering kernels for use in reactor physics applications. This generates arrays of $S(\alpha, \beta)$ values across set intervals of $\alpha, \beta$ and temperature. Thermal neutron scattering kernels required for other points are obtained through interpolation on these tables. The $S(\alpha, \beta)$ values are required in order to obtain the incoherent scattering cross sections.

$$
\sigma_{\text {incoh }}=\sigma_{\text {free }} \cdot \frac{(A+1)^{2}}{2 k T A^{2}} \sqrt{\frac{E^{\prime}}{E}} e^{-\frac{\beta}{2}} \cdot S(\alpha, \beta)
$$

where $\alpha$ is a dimensionless momentum transfer parameter given by

$$
\alpha=\frac{E^{\prime}+E-2 \mu \sqrt{E \cdot E^{\prime}}}{A k T}
$$

and $\beta$ is a dimensionless energy transfer parameter given by

$$
\beta=\frac{E^{\prime}-E}{k T}
$$

The traditional method of generating the phonon contribution to $S(\alpha, \beta)$, which is the only contribution for graphite, has been to break down the exact equation into a truncated sum of recursive convolution integrals [21].

$$
\begin{gathered}
S(\alpha, \beta)=e^{-\alpha \lambda_{s}} \sum_{n=1}^{\infty} \frac{1}{n !}\left(\alpha \lambda_{s}\right)^{n} T_{n}(\beta) \\
T_{1}(\beta)=\frac{P_{s}(\beta) \cdot e^{-\frac{\beta}{2}}}{\lambda_{s}} \\
T_{n}(\beta)=\int_{-\infty}^{\infty} T_{1}\left(\beta^{\prime}\right) \cdot T_{n-1}\left(\beta-\beta^{\prime}\right) d \beta^{\prime}
\end{gathered}
$$


where $\lambda_{s}$ is the Debye-Waller coefficient given by

$$
\lambda_{s}=\int_{-\infty}^{\infty} P_{s}(\beta) e^{-\frac{\beta}{2}} d \beta
$$

and $P_{s}$ depends on the phonon frequency spectrum $\rho(\beta)$ such that

$$
P_{s}(\beta)=\frac{\rho(\beta)}{2 \cdot \beta \cdot \sinh \left(\frac{\beta}{2}\right)}
$$

Due to small differences in the integration algorithms and evaluation methods, the thermal scattering kernels generated are not machine precision identical to those from the LEAPR module of NJOY, but the relative difference is very small, as seen in Fig. 4.3. It can been seen that the relative error in $S(\alpha, \beta)$ is on the order of $0.001 \%$ or lower for all $\beta$ (the relative error between these methods is independent of $\alpha)$.

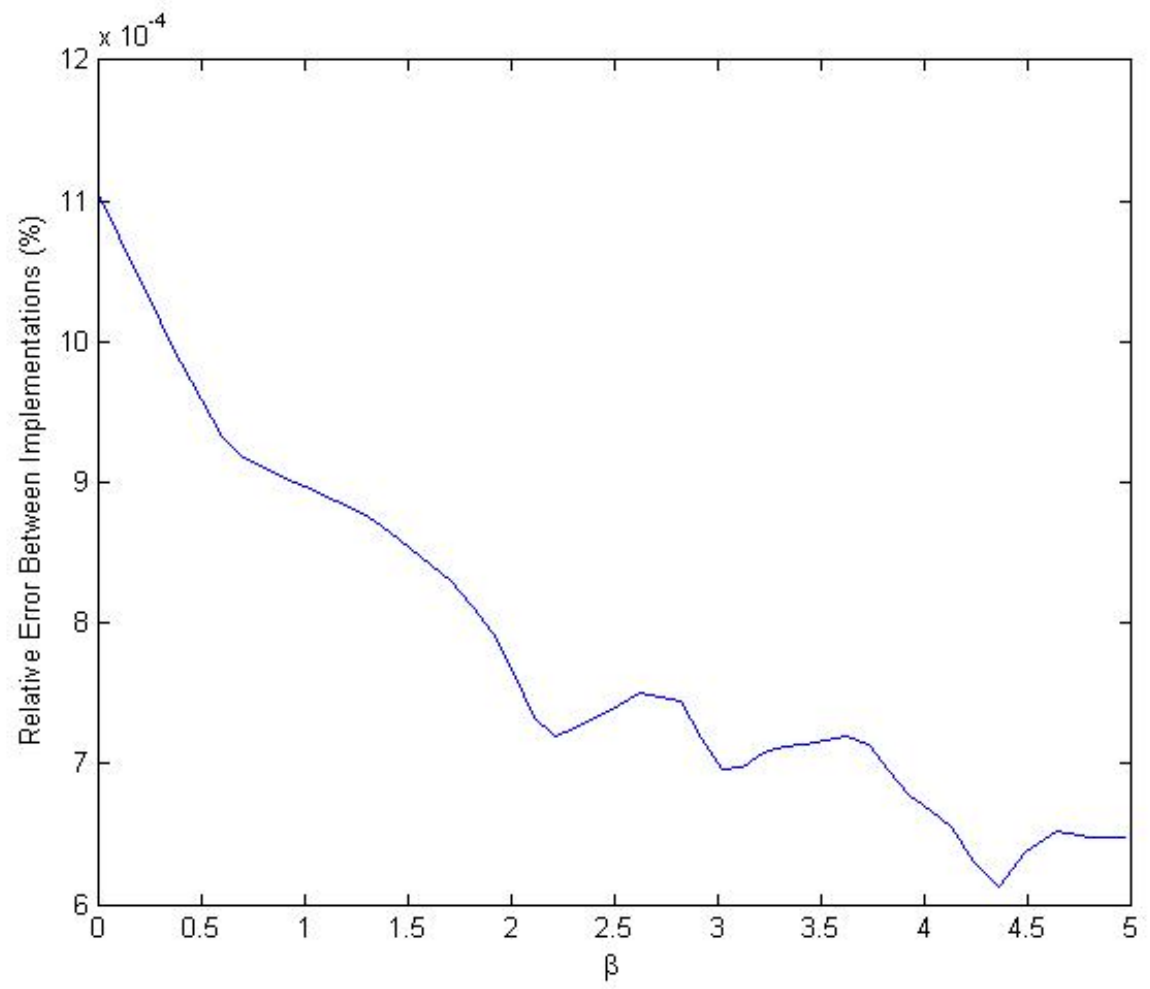

Fig. 4.3. Relative error of $\mathrm{S}(\mathrm{a}, \mathrm{b})$ kernel generated by LEAPR and OpenMC 
For complete replication of the capabilities of the LEAPR module, code for generating the coherent structure factors and Bragg energies for coherent scattering cross sections will also be required. This has not yet been developed.

\subsection{CONCLUSIONS AND FUTURE WORK}

Thus far, this research has made significant progress in improving the OpenMC Monte Carlo framework for analysis of the TREAT reactor. This project has developed a simplified model of the TREAT reactor for analysis with the OpenMC tool. Certain elements of the core have not been fully implemented, such as control rods and some detail in the structural materials, but the modular fashion in which the TREAT reactor is constructed allows for very quick setup of different patterns and configurations. Further work will be carried out to refine and validate the OpenMC TREAT model.

Due to the prevalence of graphite in the TREAT reactor and the reliance on axial streaming paths, it is extremely important that thermal neutron scattering is modelled accurately. Preliminary work indicates that the difference between bound thermal scattering and bound scattering is on the order of $800 \mathrm{pcm}$ of reactivity to the TREAT system. In order to properly predict the behavior of the reactor, sensitivity studies and uncertainty analyses will need to be carried out to determine the impact of the underlying bound thermal scattering parameters on criticality and power distribution. To this end, the pre-processing functionality of codes like NJOY for thermal neutron scattering kernels has been replicated for integration in OpenMC. This will allow a new set of cross section data to be generated internally in OpenMC when new phonon spectrum need testing. The capability for generating Bragg energies and coherent structures will also be implemented.

This method still requires internal pre-processing to generate large arrays of data at different temperature and energy values. Future work aims to move away from the old method of interpolating across tables of cross section data in both 
temperature and energy. This project has begun to examine the possibility of computing the complete contribution to the thermal scattering kernel using the first principles Fourier Transform definition for the thermal scattering kernel [5].

$$
S(\alpha, \beta)=\frac{1}{2 \pi} \int_{-\infty}^{\infty} e^{i \beta \hat{t}} \prod_{j} e^{-\left(\alpha \int_{-\infty}^{\infty} \frac{\rho_{j}(\beta)}{2 \cdot \beta \cdot \sinh \left(\frac{\beta}{2}\right)}\left[1-e^{-i \beta \hat{t}}\right] e^{-\beta / 2} d \beta\right)} d \hat{t}
$$

where the scattering kernel contribution arising from a given physical effect, $j$, is encapsulated in the frequency spectrum $\rho_{j}(\beta)$. This method will greatly assist in reducing the memory storage requirements for cross sections, although the computational efficiency of directly solving the Fourier Transforms is yet unknown.

Additionally, the coupling of OpenMC and MOOSE has been extended to model the TREAT reactor and map temperature and power distributions. Functional expansion tallies using Legendre polynomials are currently being tested for the TREAT geometry and possible extensions using other polynomial sets will be evaluated. 


\subsection{Steady State Modeling of the Minimum Critical Core of the Transient Reactor Test Facility (TREAT)}

The Transient Reactor Test Facility (TREAT) is a versatile test facility designed to physically evaluate nuclear fuels and associated structural materials in various types of excursion scenarios. It was constructed in 1958 and first went critical in 1959. The facility conducted thousands of successful experiments until 1994 when operations were suspended [22]. Historically, the immediate objective of TREAT was to provide quantitative data and visual information on the mechanisms involved with off normal conditions for reactor fuels and structural materials [22]. This information was then used to guide the development of advanced fuel designs.

The U.S. Department of Energy Office of Nuclear Energy (DOE-NE) is preparing to resume TREAT operations [22]. Historical methods for pre-transient testing involved low order computational methods. Due to the typical low accuracy of these methods, an increased number of physical calibration tests for the experiments were required. To decrease the number of these tests, a high-order modeling and simulation (M\&S) tool is being developed using MAMMOTH, the reactor physics package of the Multiphysics Object Oriented Simulation Environment (MOOSE) [23]. The goal of the work shown in this report is to develop a steady state, full core deterministic neutronics model of TREAT using the minimum critical core configuration to show cross section and mesh validity within MAMMOTH as well as identify the fundamental neutronics properties of TREAT. This work will also serve as a base point for further multiphysics model development.

\subsection{MODELING APPROACH}

A significant challenge in the modeling of TREAT is to determine how to model the streaming of neutrons in the air channel between assemblies. Recall that the diffusion coefficient in the neutron diffusion equation and the streaming term of 
the second order formulations of the transport equation have a $\frac{1}{\sigma_{t}}$ dependence. Material regions with air (very low $\sigma_{t}$ ) are difficult to develop accurate nuclear data for. In order to alleviate some of these problems, several radial homogenization schemes were developed. A complete analysis of the all homogenization models can be found in Ref [24]. In this report, two models will be discussed:

1. Full Homogenization - all radial regions are homogenized.

2. Full Fuel Homogenization with Explicit Channels - Full Homogenization with separate air cooling channels and interassembly gaps.

Models are developed in three essential steps: 1) Mesh preparation, 2) nuclear data preparation, and 3) assembly of the MAMMOTH input model. In this report, a brief description of each of these steps will be discussed. More detailed discussion for each of the parts is left for Ref. [24].

\subsection{MESH PREPARATION}

Geometric models and their corresponding meshes were created with CUBIT, a full featured mesh generation toolkit designed by Sandia National Laboratory. CUBIT is designed to create either structured or unstructured mesh elements in two- and three-dimensions [25]. A python interface is built into CUBIT allowing for a mechanism in which bodies may be created and stored. It also provides an object-oriented structure that gives users the ability to easily manipulate and query bodies. Because of these capabilities, CUBIT is often used as the mesh generation tool for MAMMOTH applications.

Models analyzed in this report utilized the Python interface within Cubit and were created using a modularized format. The following general module format was used: 1) main input; 2) geometry building functions; 3) advanced CUBIT functions; 4) main executioner. 3D models were made in the following order with their corresponding parent python module(s) enclosed in brackets: 
1. Build assemblies to exact dimensions $\{1,2\}$.

2. If core configuration, arrange assemblies via core map $\{1,4\}$.

3. "Imprint and merge" $\{4\}$.

4. Specify mesh interval refinement and mesh schemes $\{1,3,4\}$.

5. Mesh top most surfaces of geometries with 2D mesh $\{1,3,4\}$.

6. Sweep and extrude top surface mesh through rest of respective volume to produce 3D mesh $\{3,4\}$.

7. Group like elements into blocks for efficient material property application $\{1,4\}$.

8. Group outermost surface elements into sidesets for boundary condition application $\{3,4\}$.

9. Export complete model $\{4\}$.

More details on this process can be found in the following reference [24].

\subsection{CROSS SECTION DEVELOPMENT}

Cross sections used in this work were prepared primarily from SERPENT 2 using ENDF/B-VII.1 and are flux and volume weighted from full detail, 3D assembly models [26,27]. Based on previous High Temperature Reactor (HTR) studies, 26, 14, 11, and 8 group structures were evaluated [28]. It was shown that the 26 group structure produced the lowest error while the 11 and 14 group structures gave similar errors [27]. Because of this, the 11 group structure was chosen for all simulations.

Due to previously studied strong fuel-reflector interface effects in graphite reactors [26], a spatial spectral analysis for fuel assemblies was conducted within SERPENT 2. It was shown that within the fuel, large variances in the flux occur near the reflector interfaces [27]. Because of these spectral effects, the fuel region was split into two regions, an $80.97 \mathrm{~cm}$ central region surrounded by two $20 \mathrm{~cm}$ 
interfacial regions. The spectrum calculated in the reflector regions was had a more continuous flux gradient which led to the region being split into three separate regions, one $10.45 \mathrm{~cm}$ region nearest the interface, one $25.23 \mathrm{~cm}$ region in the center, and one $27 \mathrm{~cm}$ region towards the outer periphery.

\subsection{MAMMOTH MODELS}

This section shows the physical mesh, describes boundary condition treatment, and solvers used for the homogenization models tested within MAMMOTH.

\subsubsection{Standard Assemblies}

A top down view of the full radial homogenization and full fuel homogenization with explicit channels is shown in Figs. 5.1 and 5.2, respectively. The full radial homogenization model utilizes first order, 8 node hexagonal elements. The full fuel homogenization with explicit channels model utilizes first order, 8 node hexagonal elements as well as first order, 6 node wedge elements [29]. The standard axial mesh size for both models is shown in Fig. 5.3. Reflective boundary conditions were imposed on the radial sides of the models with extrapolated boundary conditions on the top and bottom.

A set of steady state calculations at different temperatures are used to determine power distribution, reaction rates, and leakage rates. For the results discussed in this report, solutions were solved with diffusion theory. Select models were solved with transport methodologies and are left for reference [5]. 

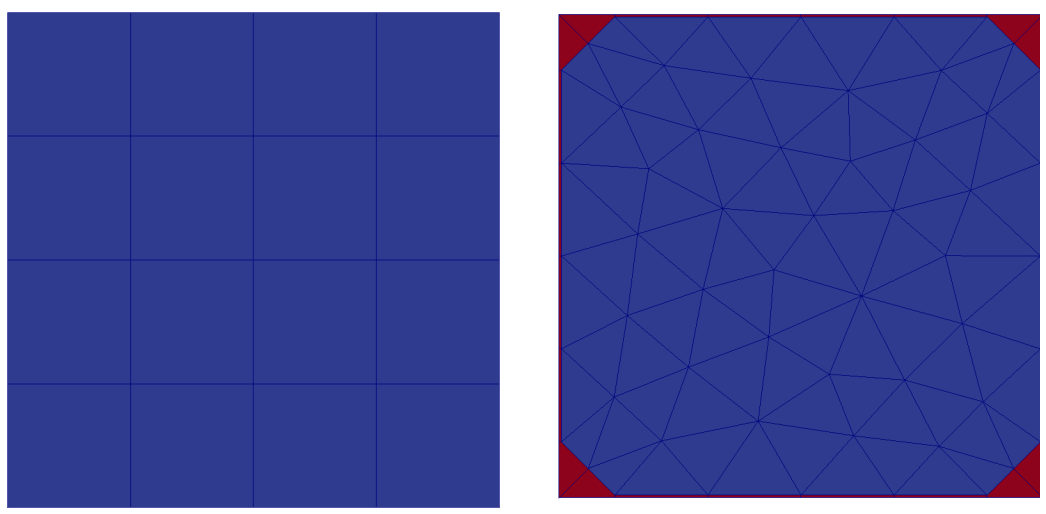

Fig 5.1. Top down view of full

Fig. 5.2. Top down view of

Fig. 5.3 Side

radial homogenization model. full fuel homogenization with explicit channels model.

view of standard axial mesh discretization.

\begin{tabular}{|c|c|c|c|c|c|c|c|c|c|c|c|c|c|c|c|c|c|c|c|}
\hline & A & B & C & D & $E$ & $F$ & G & H & J & K & L & $\mathbf{M}$ & $\mathbf{N}$ & 0 & $\mathbf{P}$ & $\mathbf{R}$ & $\mathbf{S}$ & $\mathbf{T}$ & U \\
\hline & 4 & 4 & 4 & 4 & $\overline{4}$ & 4 & 4 & 4 & 4 & 4 & 4 & 4 & 4 & 4 & 4 & 4 & 4 & 4 & 4 \\
\hline & 4 & 4 & 4 & 4 & 4 & 4 & 4 & 4 & 4 & 4 & 4 & 4 & 4 & 4 & 4 & 4 & 4 & 4 & 4 \\
\hline & A & 4 & 4 & 4 & 4 & 4 & 2 & 2 & 2 & 4 & 2 & 2 & 2 & 4 & 4 & 4 & 4 & 4 & 4 \\
\hline & 4 & 4 & 4 & 4 & 4 & 2 & 1 & 1 & 1 & 2 & 1 & 1 & 1 & 2 & 4 & 4 & 4 & 4 & 4 \\
\hline & 4 & 4 & 4 & 4 & 2 & 1 & 1 & 1 & 1 & 1 & 1 & 1 & 1 & 1 & 2 & 4 & 4 & 4 & 4 \\
\hline & 4 & 4 & 4 & 2 & 1 & 1 & 1 & 3 & 1 & 1 & 1 & 3 & 1 & 1 & 1 & 2 & 4 & 4 & 4 \\
\hline 7 & 4 & 4 & 2 & 1 & 1 & 1 & 1 & 1 & 1 & 1 & 1 & 1 & 1 & 1 & 1 & 1 & 2 & 4 & 4 \\
\hline & 4 & 4 & 2 & 1 & 1 & 3 & 1 & 1 & 1 & 1 & 1 & 1 & 1 & 3 & 1 & 1 & 2 & 4 & 4 \\
\hline & 4 & 4 & 2 & 1 & 1 & 1 & 1 & 1 & 1 & 1 & 1 & 1 & 1 & 1 & 1 & 1 & 2 & 4 & 4 \\
\hline & 4 & 4 & 4 & 2 & 1 & 1 & 1 & 1 & 1 & 1 & 1 & 1 & 1 & 1 & 1 & 2 & 4 & 4 & 4 \\
\hline & 4 & 4 & 2 & 1 & 1 & 1 & 1 & 1 & 1 & 1 & 1 & 1 & 1 & 1 & 1 & 1 & 2 & 4 & 4 \\
\hline & 4 & 4 & 2 & 1 & 1 & 3 & 1 & 1 & 1 & 1 & 1 & 1 & 1 & 3 & 1 & 1 & 2 & 4 & 4 \\
\hline & 4 & 4 & 2 & 1 & 1 & 1 & 1 & 1 & 1 & 1 & 1 & 1 & 1 & 1 & 1 & 1 & 2 & 4 & 4 \\
\hline & 4 & 4 & 4 & 2 & 1 & 1 & 1 & 3 & 1 & 1 & 1 & 3 & 1 & 1 & 1 & 2 & 4 & 4 & 4 \\
\hline & 4 & 4 & 4 & 4 & 2 & 1 & 1 & 1 & 1 & 1 & 1 & 1 & 1 & 1 & 2 & 4 & 4 & 4 & 4 \\
\hline & 4 & 4 & 4 & 4 & 4 & 2 & 1 & 1 & 1 & 2 & 1 & 1 & 1 & 2 & 4 & 4 & 4 & 4 & 4 \\
\hline & 4 & 4 & 4 & 4 & 4 & 4 & 2 & 2 & 2 & 4 & 2 & 2 & 2 & 4 & 4 & 4 & 4 & 4 & 4 \\
\hline & 4 & 4 & 4 & 4 & 4 & 4 & 4 & 4 & 4 & 4 & 4 & 4 & 4 & 4 & 4 & 4 & 4 & 4 & 4 \\
\hline & 4 & 4 & 4 & 4 & 4 & 4 & 4 & 4 & 4 & 4 & 4 & 4 & 4 & 4 & 4 & 4 & 4 & 4 & 4 \\
\hline
\end{tabular}

Fuel Assembly

Zr Clad Dummy Assembly

Control Assembly

Al Clad Dummy Assembly

Fig. 5.4. Minimum critical core configuration. 


\subsubsection{Minimum Critical Core}

The core configuration for the minimum critical core is shown in Fig. 5.4. It consists of 138 fuel assemblies, 8 control assemblies, 40 Zirconium clad reflector assemblies, and 175 Aluminum clad reflector assemblies. It was built to each of the various radial homogenization schemes and axially partitioned identical to the standard assembly models. Extrapolated vacuum boundary conditions were applied to each exterior surface.

Figure 5.5 shows a one-quarter top down view of the meshed core with full radial homogenization. In this scheme only first order hexagonal elements were used. Figure 5.6 shows a one-quarter top down view of the meshed core with full fuel homogenization with explicit inter-assembly gaps and cooling channels. First order hexagonal and wedge elements are used in this configuration.

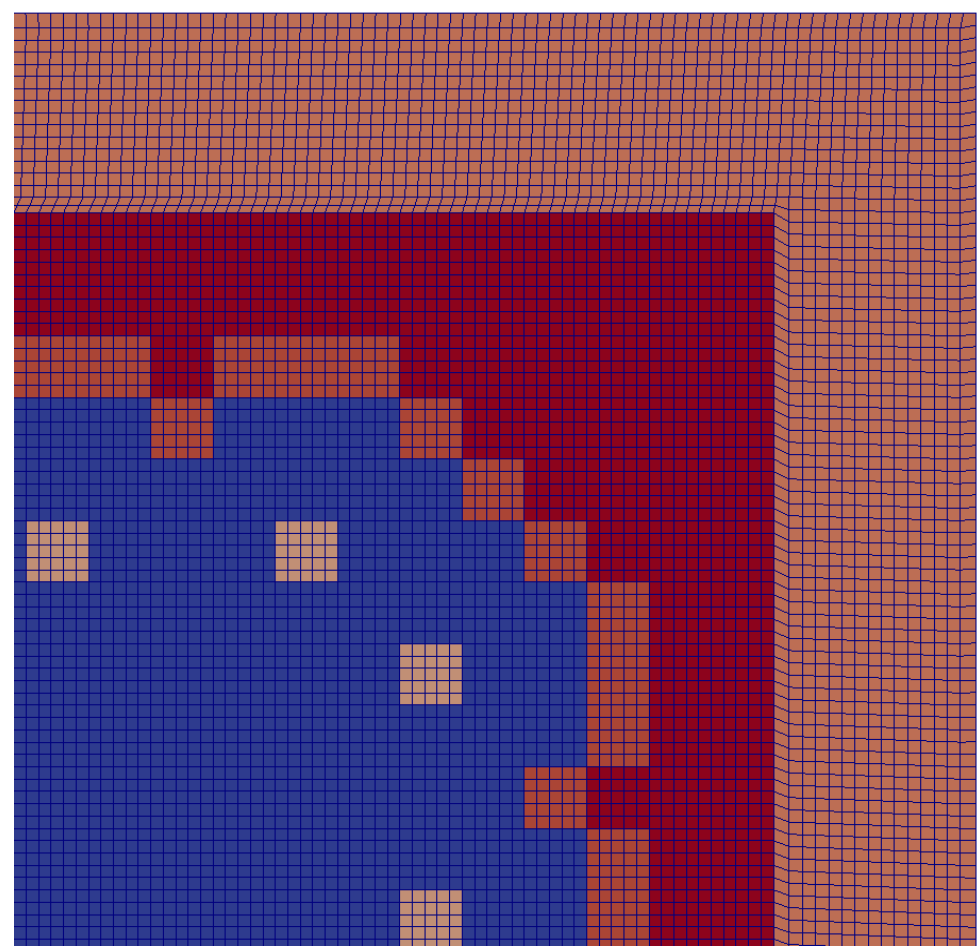

Fig. 5.5. One-quarter top down view of full radial homogenization for the minimum critical core. 


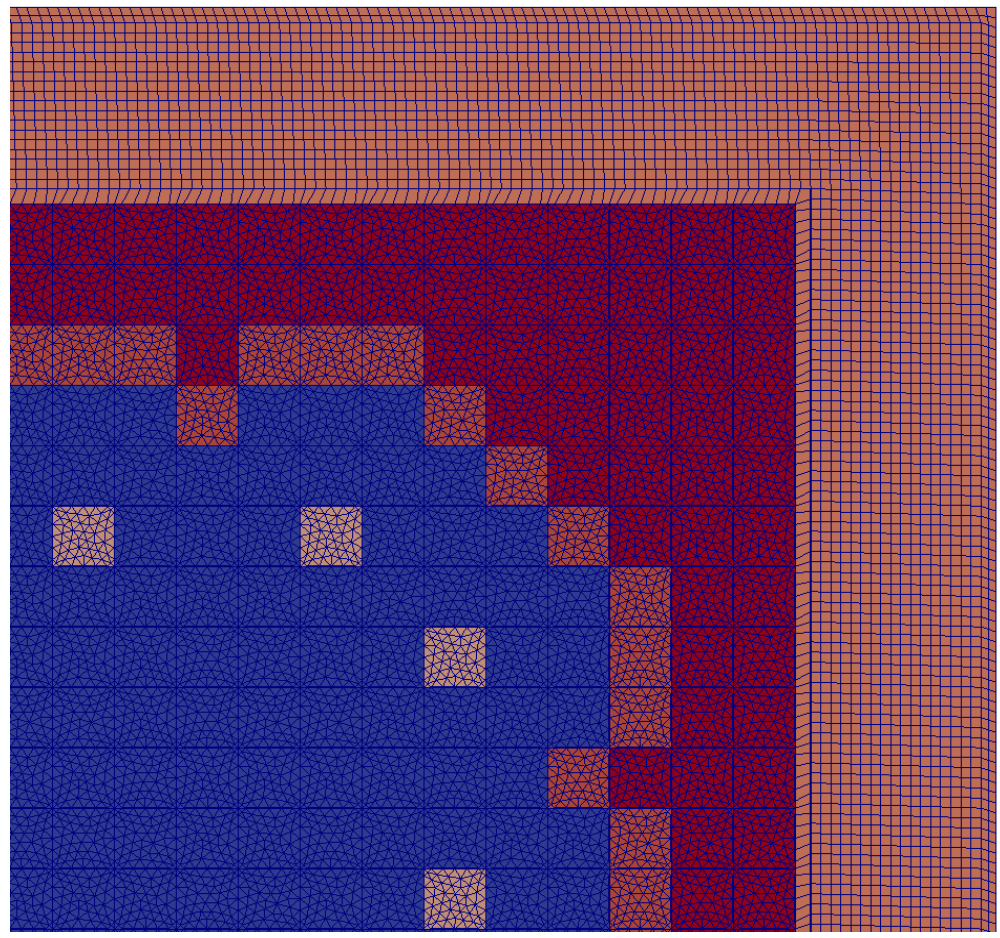

Fig. 5.6. One-quarter top down view of full fuel homogenization with explicit channels for the minimum critical core.

\subsection{RESULTS}

Because MAMMOTH is largely under development, it is imperative to show that model results agree with reference Monte Carlo solutions. Assembly models will be evaluated on axial power distributions, $k_{e f f}$, and integral reaction rates. Full core models will be evaluated on the following: 1) axially integrated, radial power distributions; 2) radially averaged, axial power distributions; 3) $k_{\text {eff }}$; and 4) integral reaction rates. All simulations are normalized to an arbitrary power of $2000 \mathrm{~W}$. It is important to note that the value of $k_{e f f}$ is a balance between the production, absorption, and leakage rates (Eq. 5.1).

$$
k_{\text {eff }}=\frac{\text { production rate }}{\text { leakage rate }+ \text { absoprtion rate }}
$$


Since $k_{e f f}$ is an integral parameter, it is difficult to determine the extent of cancellation of error between over prediction and under prediction of respective reaction rates when compared to reference results. Therefore it is necessary to quantify specific integral reaction rates (i.e. fission source, capture, and leakage rates).

\subsubsection{Single Assembly, Full Homogeneous Model}

Reference reaction rates can be found in Section 6.1 of [24]. Figure 5.6 shows the calculated power distribution for the full radial homogenization model.

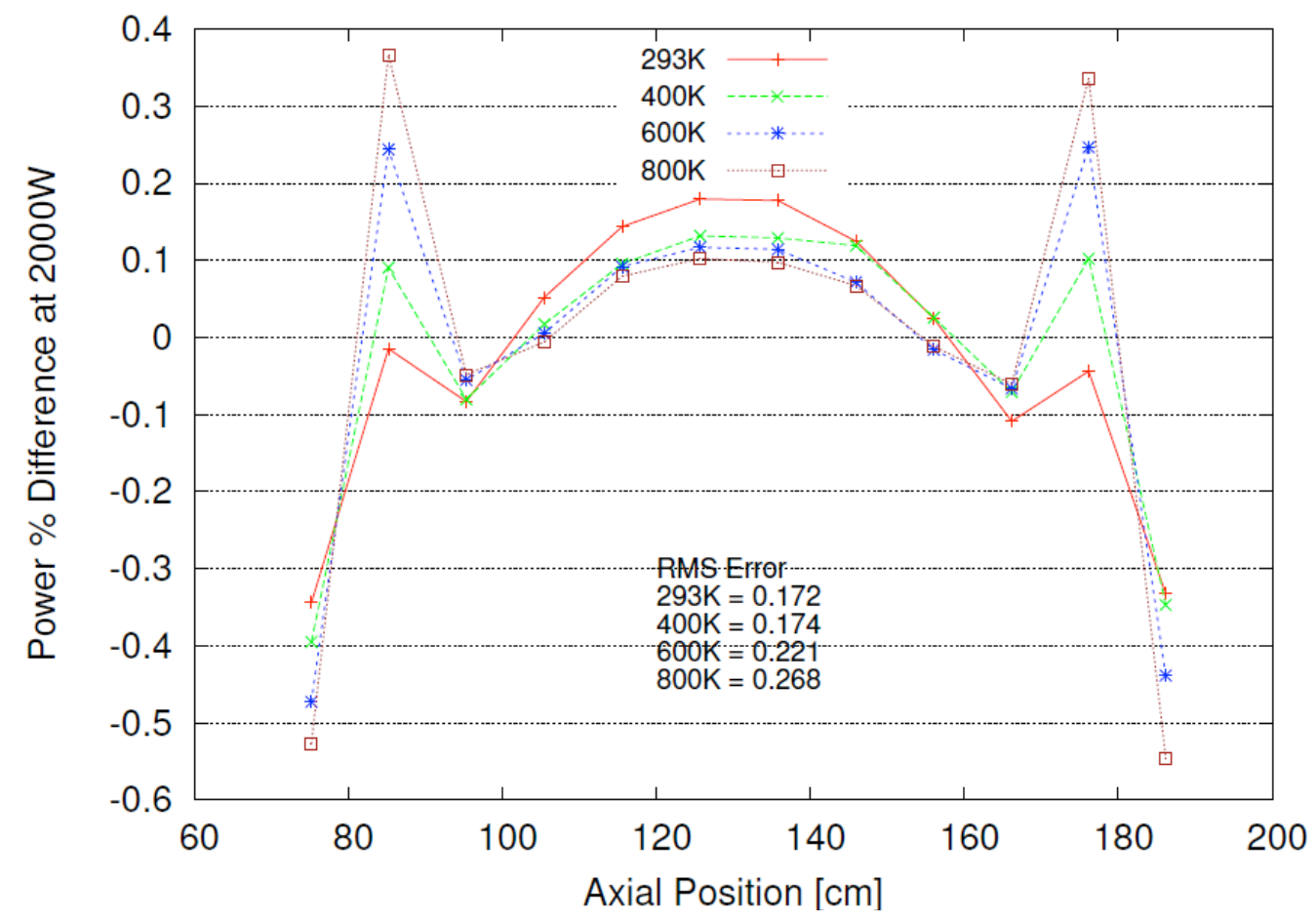

Fig. 5.6. Full homogeneous model, axial power distribution at varying temperatures.

It can be seen that the RMS error increases as a function of temperature with the highest level of uncertainty occurring at the periphery of the assembly. The reason for this is due to the fact that cross sections at the periphery are weighted over a larger flux gradient. By having a larger flux distribution in this weighting, spatial fidelity of cross sections are diminished. 
Results for the diffusion solver for the fully homogenized model show a significant over prediction of the eigenvalue when compared to the reference SERPENT calculation (Table 5.1). The overall contribution of the capture and leakage reaction rates for this model were found to be $38.29 \%$ and $2.89 \%$ at $293 \mathrm{~K}$ and increasing to $40.40 \%$ and $3.33 \%$ at $800 \mathrm{~K}$, confirming experimentally observed spectral hardening. Based on Equation 1, the reason for the larger over prediction of the calculated eigenvalue is due to the under prediction of the capture and leakage rates. Spatial mesh convergence and transport analysis are left for reference [24].

Table 5.1. Calculated reaction rates and eigenvalues for standard assembly, full radial homogeneous model.

\begin{tabular}{|c|c|c|c|c|}
\hline Temp $(K)$ & $k_{\text {eff }}(\mathrm{pcm})$ & $\begin{array}{c}\text { Diff Fission } \\
\text { Source Rate } \\
(\%)\end{array}$ & $\begin{array}{c}\text { Diff Capture } \\
\text { Rate (\%) }\end{array}$ & $\begin{array}{c}\text { Diff Leakage } \\
\text { Rate (\%) }\end{array}$ \\
\hline 293 & $\begin{array}{c}1.43342 \\
(582.84)\end{array}$ & -0.829 & -0.529 & -17.966 \\
\hline 400 & $\begin{array}{l}1.41837 \\
(631.98)\end{array}$ & -0.885 & -0.590 & -18.262 \\
\hline 600 & $\begin{array}{l}1.39242 \\
(695.10)\end{array}$ & -0.968 & -0.688 & -17.951 \\
\hline 800 & $\begin{array}{l}1.37123 \\
(756.71)\end{array}$ & -1.027 & -0.750 & -18.065 \\
\hline
\end{tabular}

\subsubsection{Single Assembly, Full Fuel Homogenization with Explicit Channels Model}

In order to properly model the explicit cooling channels and interassembly gaps of this homogenization, a study on diffusion treatment was completed. It was found that by artificially adjusting the group wise diffusion coefficient by a factor of 2.5 , the most accurate power distributions were obtained [24]. 
Figure 7 shows the calculated power distribution as a function of temperature for this homogenization. It can be seen that by explicitly modeling the cooling channels and interassembly gaps, significant improvements over the fully homogeneous model are made. As before, the increased error in the peripheries of the fuel is primarily caused by flux weighting of the cross sections.

Table 2 shows the integral reaction rates for this homogenization scheme. It can be seen that improvements were made in the fission source rate and leakage rate calculations when compared to that of Table 1. Because of these improvements and the simultaneous significant improvement in power distribution, it can be inferred that the reaction rate error is driven by uncertainty within the reflector region and that reaction rates within the fuel are well preserved. Better preserving reaction rates within the reflector regions is reserved for future work. Spatial convergence is shown in [24].

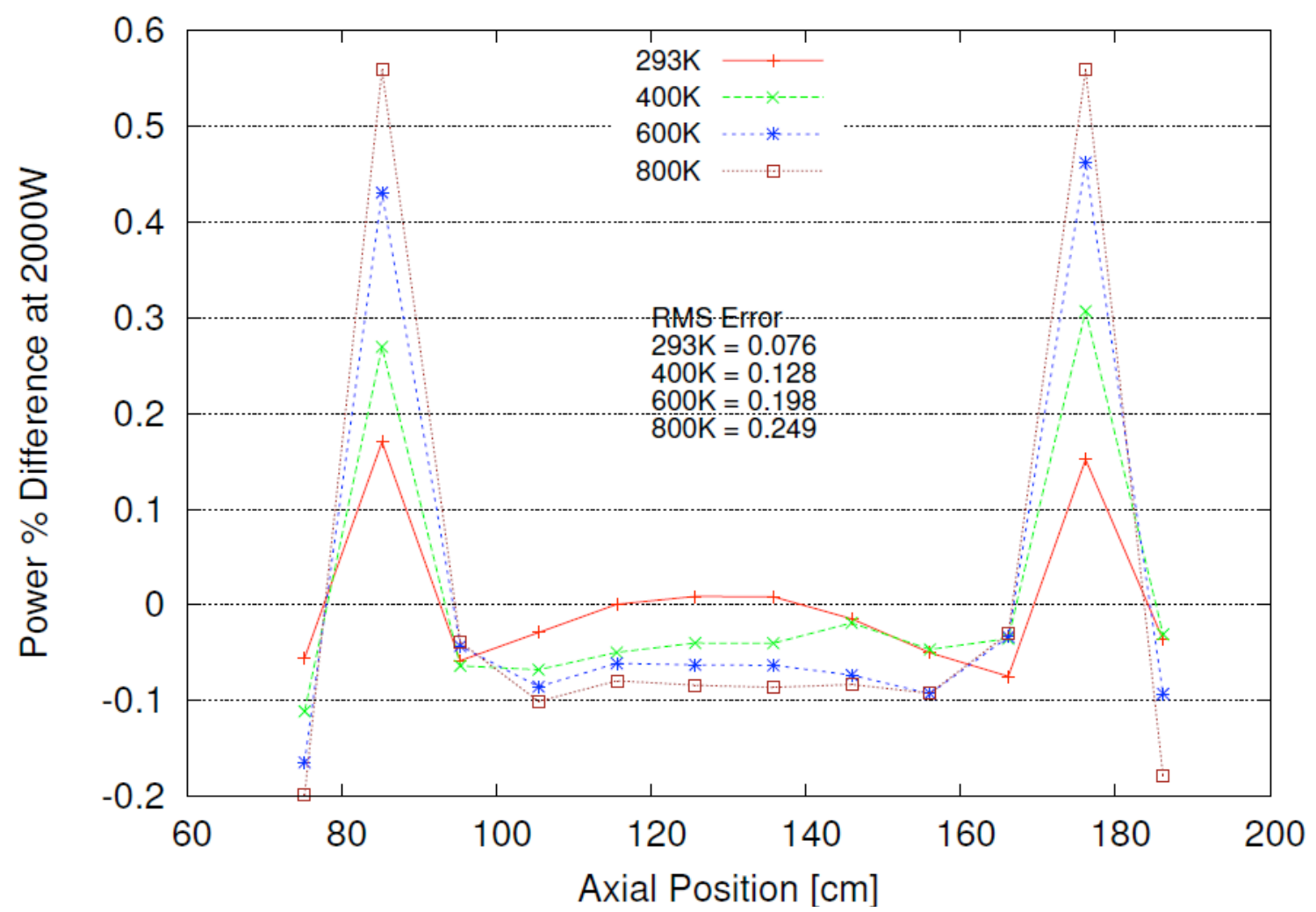

Fig 5.7. Axial power distribution at varying temperatures for full fuel homogenization with explicit channels model. 
Table 5.2 Calculated reaction rates and eigenvalues for standard assembly, full fuel homogenization with explicit channels model.

\begin{tabular}{|c|c|c|c|c|}
\hline Temp $(K)$ & $\begin{array}{c}k_{\text {eff }} \\
(p c m)\end{array}$ & $\begin{array}{c}\text { Diff Fission } \\
\text { Source Rate } \\
(\%)\end{array}$ & $\begin{array}{c}\text { Diff Capture } \\
\text { Rate (\%) }\end{array}$ & $\begin{array}{c}\text { Diff Leakage } \\
\text { Rate (\%) }\end{array}$ \\
\hline 293 & $\begin{array}{r}1.43342 \\
(582.84)\end{array}$ & -0.723 & -0.586 & -16.605 \\
\hline 400 & $\begin{array}{r}1.41837 \\
(631.98)\end{array}$ & -0.776 & -0.643 & -16.930 \\
\hline 600 & $\begin{array}{r}1.39242 \\
(695.10)\end{array}$ & -0.852 & -0.741 & -16.700 \\
\hline 800 & $\begin{array}{r}1.37123 \\
(756.71)\end{array}$ & -0.912 & -0.800 & -16.807 \\
\hline
\end{tabular}

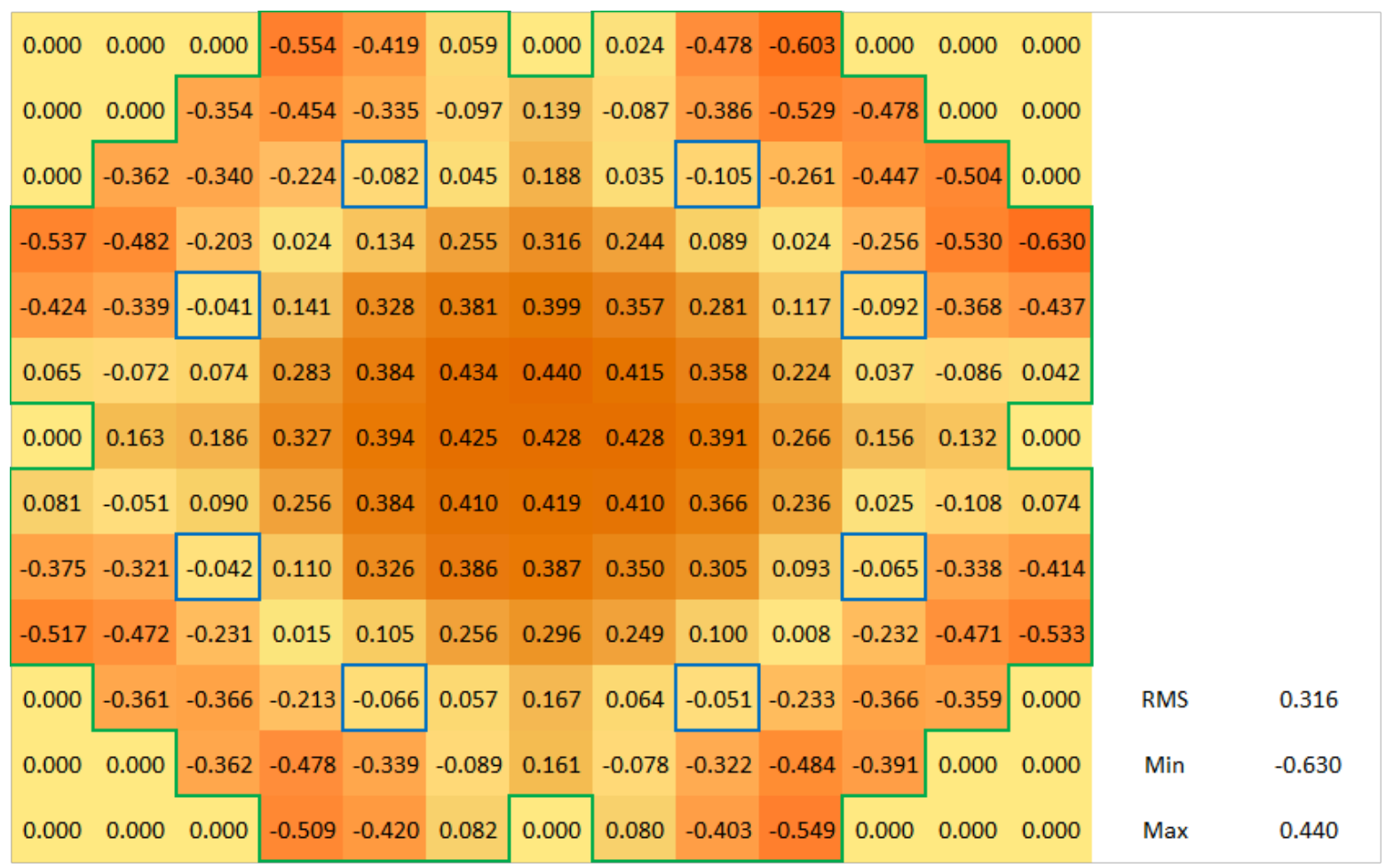

Fig. 5.8. Axially integrated, radial power distribution (RPD) at $293 \mathrm{~K}$ for minimum critical core, full homogenization model. 


\subsubsection{Minimum Critical Core, Full Homogeneous Model}

Performance of minimum critical core models were based on axially integrated radial power distributions, radially integrated axial power distributions, reaction rates, and eigenvalue calculations. Reference reaction rates can be found in Section 6.2 of [5]. Figure 5.8 shows the MAMMOTH calculated axially integrated radial power distribution at $293 \mathrm{~K}$ for the full radial homogenization model.

RMS error reported for the RPD is $0.316 \%$ with a minimum and maximum uncertainty of $-0.630 \%$ and $0.440 \%$, respectively. A discussion on the error propagation across the core is discussed within [24].

Figure 5.9 shows the radially integrated axial power. The lowest error for each temperature case occurs in the center of the core with the error increasing at the peripheries due to the increased flux gradient.

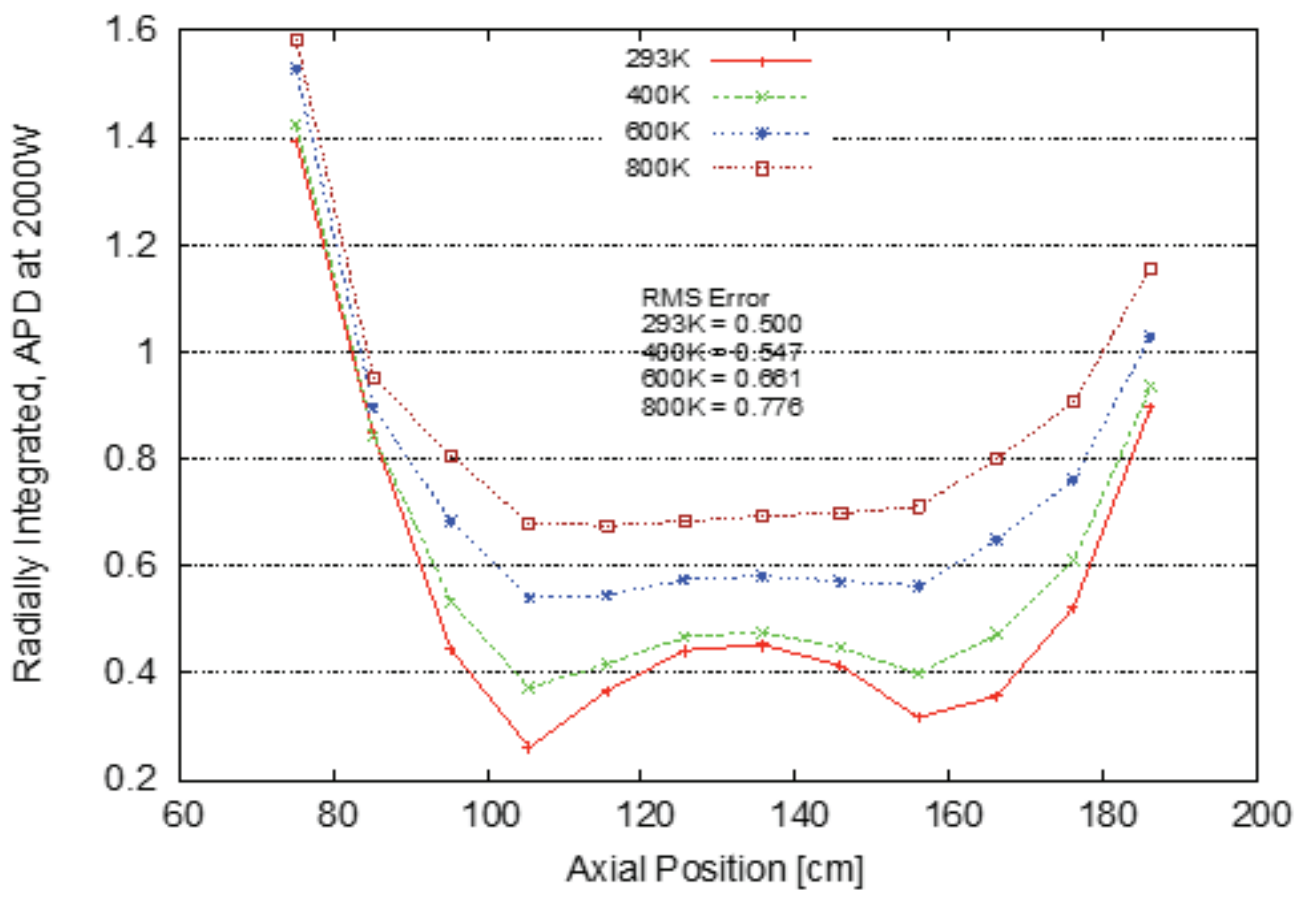

Fig. 5.9. Radially integrated, axial power distribution at $293 \mathrm{~K}$ for minimum critical core, full homogenization model. 
Table 5.3 shows the calculated reaction rates for the fully homogenized model. It can be seen that this homogenization largely over predicts the eigenvalue reported by reference SERPENT solutions. The reason for this large over prediction is due to the large under prediction of integral captures. Because the power distribution is relatively accurate, it can be inferred that the mis-prediction of capture reactions within the axial and radial reflectors are the driving source of uncertainty.

Table 5.3. Calculated reaction rates and eigenvalues for minimum critical core, full radial homogenization model.

\begin{tabular}{|c|c|c|c|c|}
\hline Temp (K) & $\begin{array}{c}k_{\text {eff }} \\
(p \mathrm{~cm})\end{array}$ & $\begin{array}{c}\text { Diff Fission } \\
\text { Source Rate } \\
(\%)\end{array}$ & $\begin{array}{c}\text { Diff Capture } \\
\text { Rate (\%) }\end{array}$ & $\begin{array}{c}\text { Diff Leakage } \\
\text { Rate (\%) }\end{array}$ \\
\hline 293 & $\begin{array}{c}1.01961 \\
(1388.79)\end{array}$ & -1.407 & -13.518 & 33.963 \\
\hline 400 & $\begin{array}{c}0.99509 \\
(1509.00)\end{array}$ & -1.498 & -13.339 & 31.440 \\
\hline 600 & $\begin{array}{c}0.95590 \\
(1710.37)\end{array}$ & -1.630 & -13.723 & 31.392 \\
\hline 800 & $\begin{array}{c}0.92462 \\
(1879.18)\end{array}$ & -1.732 & -14.034 & 31.429 \\
\hline
\end{tabular}

\subsubsection{Minimum Critical Core, Full Fuel Homogenization with Explicit Channels Model}

In this model, all of the cooling channels, interassembly gaps, and the gap between the CP-2 permanent reflector and core are treated with a diffusion coefficient of 2.5 [24]. Figure 5.10 shows the axially integrated RPD for the full fuel homogenization with explicit channels model.

By explicitly modeling the channels and gaps, the RMS in the RPD is improved by $29.7 \%$. A discussion on error distribution across the core is left for reference [24]. Figure 5.11 shows the radially integrated axial power distribution for this homogenization. The reported RMS values in Figure 10 are higher than those reported in Figure 8 for the full radial homogenization. However, the RMS within 
the central fuel region of Figure 10 is more accurate than that of Figure 8 by $1.55 \%$, confirming observable improvements by explicitly modeling the cooling channel and inter-assembly gaps. The reason for the increase in total RMS error is due to control assembly modeling [24].

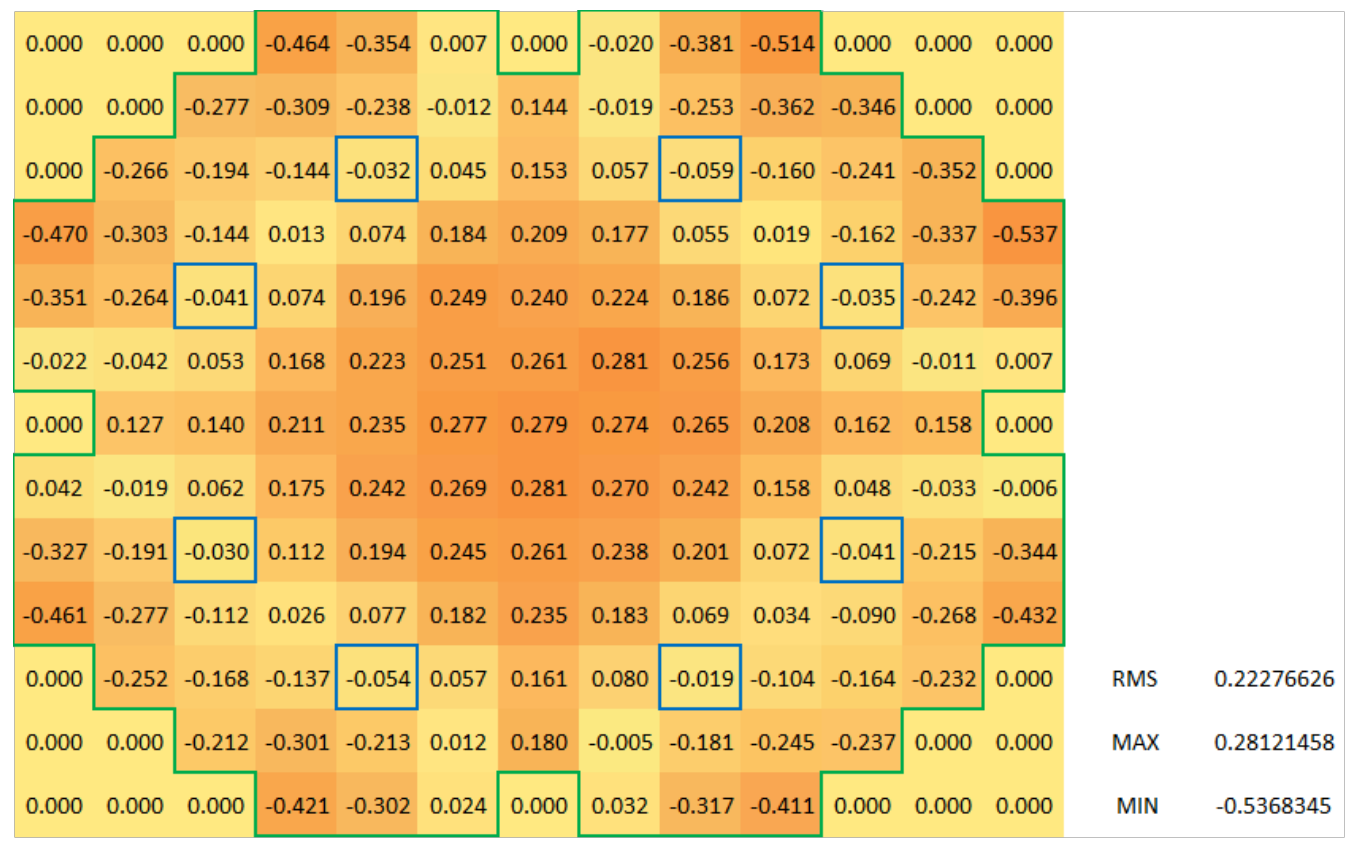

Fig. 5.10. Axially integrated, radial power distribution at $293 \mathrm{~K}$ for full fuel homogenization with explicit channels model.

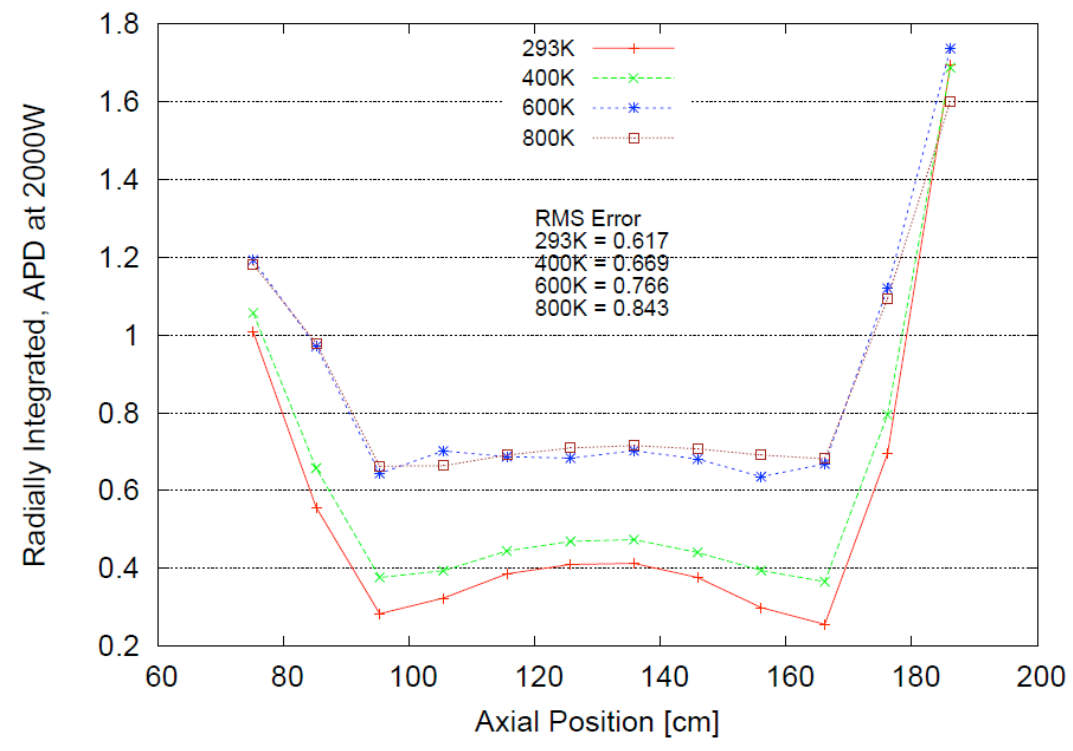

Fig. 5.11. Radially integrated, axial power distribution at $293 \mathrm{~K}$ for minimum critical core, full fuel homogenization with explicit channels model. 
Table 5.4 shows the associated reaction rates and eigenvalue for the full fuel homogenization and explicit channels core. It can be seen that the eigenvalue is significantly improved when compared to that of the results of Table 5.3. The reason for this improvement however is simply due to a cancellation of error with the capture and leakage rates [24].

Table 5.4 Calculated reaction rates and eigenvalues for minimum critical core, full fuel homogenization with explicit channels model.

\begin{tabular}{|c|c|c|c|c|}
\hline Temp (K) & $\begin{array}{c}k_{\text {eff }} \\
(p c m)\end{array}$ & $\begin{array}{c}\text { Diff Fission } \\
\text { Source Rate } \\
(\%)\end{array}$ & $\begin{array}{c}\text { Diff Capture } \\
\text { Rate (\%) }\end{array}$ & $\begin{array}{c}\text { Diff Leakage } \\
\text { Rate (\%) }\end{array}$ \\
\hline 293 & $\begin{array}{c}1.00575 \\
(24.68)\end{array}$ & -0.056 & -12.180 & 40.195 \\
\hline 400 & $\begin{array}{c}0.98104 \\
(73.21)\end{array}$ & -0.095 & -12.402 & 40.149 \\
\hline 600 & $\begin{array}{c}0.94159 \\
(131.24)\end{array}$ & -0.128 & -12.746 & 40.181 \\
\hline 800 & $\begin{array}{c}0.91019 \\
(166.12)\end{array}$ & -0.172 & -12.996 & 40.168 \\
\hline
\end{tabular}

\subsection{Future Work}

A common trend observed in all of the homogenization models, regardless of whether it was a single assembly or core calculation, was the inability to accurately calculate the integral leakage rate. This is due to a lack of flux distribution accuracy within the reflector regions and the current inability to calculate accurate diffusion coefficients for near void regions.

To obtain improved flux distributions within the reflector regions, future work involves developing super-homogenized (SPH) corrected cross sections. A more detailed discussion of the methodology of SPH corrected cross sections and how they will be used in this work is left for reference $[24,27,30]$. 
It has been shown that the isotropic diffusion coefficients used in this work are insufficient in capturing the diffusive characteristics of the TREAT core. Future work will involve developing ways to calculate anisotropic, or directional, diffusion coefficients. Two separate alternative methods for obtaining anisotropic diffusion coefficients are currently under development. The first of these methods involves developing a high fidelity, first order transport solve within MAMMOTH and equating the quotient of the calculated current and flux to the diffusion coefficient through Fick's Second Law (Eq. 5.2) [31].

$$
\boldsymbol{D}_{\boldsymbol{k}}=\frac{\boldsymbol{J}_{\boldsymbol{k}}}{\nabla \phi_{k}} ; \quad k=x, y, z
$$

The second methodology currently under development deals with collaboration with the Massachusetts Institute of Technology (MIT) and OpenMC. At the time of this writing, INL and MIT are currently working together to develop this capability for TREAT applications.

In this work it was shown that the uncertainty was driven by the modeling of the control assembly. In order to allow for these results to be viable for future transient work, it will be imperative that the control assemblies are more accurately modeled in future work. At the time of this writing, preliminary work is being completed on single control assembly calculations as well as 5x5 "minicores" to study interassembly interactions.

\subsection{CONCLUSIONS}

The main objectives of this work were to quantify various neutronics parameters for the minimum critical core configuration of the Transient Reactor Test Facility (TREAT). These parameters were calculated using the reactor physics package MAMMOTH within the MOOSE framework. Cross sections used in this work were developed with SERPENT 2 using ENDF/B-VII. 1 cross sections and are flux and volume weighted from full detail, 3D assembly models. For single assembly calculations, axial power distributions, eigenvalues, and reaction rates 
were figures of merit. For the full core calculations, axially integrated radial power distributions, radially integrated axial power distributions, eigenvalues, and reaction rates were figures of merit. All MAMMOTH calculated results were compared to reference SERPENT solutions.

It was shown for the single assembly calculations that by explicitly modeling the cooling channels and interassembly gaps, calculated power distributions are markedly improved over that of the fully homogenized model. The explicit cooling channel and interassembly gap model produced an RMS APD of $0.076 \%$. Inaccuracies in single assembly calculations were determined to be due to inaccurate representations of the flux shape and diffusion coefficient treatment in the reflector regions.

Core configurations also produced enhanced accuracy when cooling channels and interassembly gaps were explicitly modeled. Axially integrated, radial power distributions reported uncertainties of $0.223 \%$, a $29.43 \%$ improvement over that of the full radial homogenization. However, the integral reaction rates for this model were inaccurate. As was discussed, because of the accurate power distribution, it can be inferred that the inaccuracies shown stem from inaccurate flux distributions within the radial and axial reflector regions.

Future work will involve a more detailed analysis of diffusion coefficient treatment though anisotropic diffusion coefficients as well as better preserving the flux distributions within reflector regions. Efforts in both of these are currently being undertaken at the time of this writing. It is expected that with these improvements, all of the target quantifications will improve when compared to reference solutions. 


\subsection{CONCLUSIONS}

All four of these research projects have successfully met their intended goals and enhanced INL modeling and simulation efforts. This work afforded internship opportunities for summer appointments at INL for both Zachary Prince (TAMU) and Anthony Alberti (OSU) at INL, working with the reactor physics team. It also provided the basis for Mr. Alberti to complete the requirements for a Master's Degree from Oregon State University. It provided first year research for Mr. Mausolff, who started his graduate research this Fall. And it provided continued support for Ellis and Haugen, who are continuing their $\mathrm{PhD}$ research at MIT, expecting to complete in 2016 and 2017, respectively. Continued support for MIT, TAMU, and UF is planned for FY16. TREAT-related research will be continued, along a more advanced line, for Mr. Alberti's PhD research. However, the NEAMS PI has identifed an alternate line of funding for this research for FY16. 


\subsection{REFERENCES}

1. Argonne Computing Center. "Benchmark problem book." $A N L-7416$, Suppl. 2 (1977).

2. C.L. BENTLEY, "Improvements in a Hybrid Stochastic/Deterministic Method for Transient, Three-Dimensional Neutron Transport," Ph.D. Dissertation, Nuclear Engineering Department, The University of Tennessee, Knoxville (December 1996)

3. K.L.GOLUOGLU, "Revitalization and Status of the Hybrid Stochastic/Deterministic Computer Code for Transient, ThreeDimensional Neutron Transport, TDKENO," A practice project for Master of Science degree, The University of Tennessee, Knoxville (2004).

4. S. GOLUOGLU et al., "TDKENO: A Hybrid Time-dependent Transport Analysis Tool",

5. S. GOLUOGLU L. M. PETRIE, et al., "Monte Carlo Criticality Methods and Analysis Capabilities in SCALE," Technical Paper for Nuclear Technology - SCALE 6 Special Issue, (Oct 2010).

6. S.M. BOWMAN, "KENO-VI Primer: A Primer for Criticality Calculations with SCALE/KENO-VI Using GeeWiz," Oak Ridge National Laboratory, Oak Ridge TN. ORNL/TM-2008/069 (2008).

7. R.D. BUSCH, "KENO Va Primer: A Primer for Criticality Calculations with SCALE/KENO Va Using CSPAN for Input.," No. ORNL/TM2002/155. ORNL Oak Ridge National Laboratory (US) (2003).

8. G.A. FREUND et al., "Design Summary Report on the Transient Reactor Test Facility", ANL-6034, (July 1960).

9. W.R. ROBINSON and T.H. BAUER, "The M8CAL Power Calibration Experiment," ANL-IFR-212, http://www.osti.gov/scitech/servlets/purl/1130413 (May 1994).

10. R. SANCHEZ and N. J. MCCORMICK, "A Review of Neutron Transport Approximations," Nuclear Science and Engineering, 80, 481-535, (1982).

11. D. GASTON, C. NEWMAN and D. LEBRUN-GRANDIE. "Moose: A parallel computational framework for coupled systems of nonlinear equations.” Nucl. Engrg. Design, 239: pp. 1768 - 1778 (2009).

12. K. OTT. "Quasi-static treatment of spatial phenomena in reactor dynamics." Nuclear Science and Engineering, 26: p. 563 (1966).

13. S. DULLA, E. H. MUND, and P. RAVETTO. "The quasi-static method revisited." Progress in Nuclear Energy, 50(8): pp. 908 - 920 (2008). 
14. J. B. YASINSKY. "Some numerical experiments concerning space time reactor kinetics behavior." Nuclear Science and Engineering, 22: p. 171 (1965).

15. L. HAGEMAN and J. YASINSKY. "Comparison of alternating direction time differencing method with other implicit method for the solution of the neutron group diffusion equations." Nucl. Sci. Eng., 38: pp. 8 - 32 (1969).

16. S. LANGENBUCH and W. WERNER. "Coarse- mesh flux-expansion method for the analysis of space- time effects in large light water reactor cores.” Nucl. Sci. Eng., 63: pp. 437 - 456 (1969).

17. D. R. MACFARLANE J. D. GEIER G. A. FREUND, P. ELIAS and J. F. BOLAND. Design summary report on the transient reactor test facility. Technical Report ANL- 6034, Argonne National Laboratory, 1960.

18. D. P. GRIESHEIMER. Functional Expansion Tallies for Monte Carlo Simulations. PhD thesis, The University of Michigan, 2005.

19. M. ELLIS, D. GASTON, B. FORGET, and K. SMITH. "Preliminary coupling of the Monte Carlo code OpenMC and the Multiphysics ObjectOriented Simulation Environment (MOOSE) for Analyzing Doppler Feedback in Monte Carlo Simulations. ANS MC2015 - Joint International Conference on Mathematics and Computation (M\&C), Supercomputing in Nuclear Applications (SNA) and the Monte Carlo (MC) Method, April 2015.

20. R. W. SWANSON and L. J. HARRISON. The e ect of carbon crystal structure on treat reactor physics calculations. Proceedings of the 1988 International reactor physics conference, 4, September 1988.

21. NJOY99.0 Manual, 2000. PSR-480.

22. U.S. DEPARTMENT OF ENERGY, "Mission Need Statement for the Resumption of Transient Fuel Testing”, U.S. DOE, December 3 ${ }^{\text {rd }}, 2010$.

23. J. ORTENSI, et al., "Full Core TREAT Kinetics Demonstration Using Rattlesnake/BISON Coupling Within MAMMOTH - INL/EXT-1536268", Idaho National Laboratory, August 2015.

24. A. L. ALBERTI, "Steady State Modeling of the Minimum Critical Core of the Transient Reactor Test Facility", Thesis, Oregon State University, 2015.

25. R. MORRIS, “CUBIT 15.0 User Documentation”, ETI, UT, 2014.

26. J. LEPPÄNEN, “Serpent progress report 2012”, VTT-R-00300-14, VTT Technical Research Centre of Finland, 2014.

27. J. ORTENSI, et al., "Methodologies and Requirements for the Generation of Physics Data Inputs to MAMMOTH Transient Simulations in Support of the Transient Reactor Test Facility", Technical Report INL/EXT-1536265, Idaho National Laboratory, September 2015. 
28. INTERNATIONAL ATOMIC ENERGY AGENCY, "Evaluation of High Temperature Gas Cooled Reactor Performance: Benchmark Analysis Related to Initial Testing of the GTTR and HTR-10, IAEA-TECDOC$13821 ", 2003$.

29. L. A. SCHOOF and V. R. YARBERRY, "EXODUS II: A Finite Element Data Model, SAND92-2137", Sandia National Laboratory, December, 1995.

30. A. HERBERT and G. MATHONNIERE, "Development of a ThirdGeneration Superhomgeneisation Method for the Homogenization of a Pressurized Water Reactor Assembly", Commissariat a l'Energie Atomique, Cedex, France, April 12 $2^{\text {th }}, 1993$.

31. M. D. DeHART, S SCHUNERT, "Rattlesnake Updates to Improve TREAT Simulation Capabilities INL/MIS-15-36598”, Idaho National Laboratory, 2015. 